\title{
Existence results for multi-term fractional differential equations with nonlocal multi-point and multi-strip boundary conditions
}

\author{
Ravi P. Agarwal ${ }^{1,2,3}$, Ahmed Alsaedi ${ }^{3}$, Najla Alghamdi ${ }^{3,4}$, Sotiris K. Ntouyas ${ }^{3,5}$ and Bashir Ahmad ${ }^{3 *}$ (D)
}

"Correspondence:

bashirahmad_qau@yahoo.com ${ }^{3}$ Nonlinear Analysis and Applied Mathematics (NAAM) - Research Group, Department of Mathematics, Faculty of Science, King Abdulaziz University, Jeddah, Saudi Arabia Full list of author information is available at the end of the article

\begin{abstract}
In this paper, we discuss the existence and uniqueness of solutions for a new class of multi-point and multi-strip boundary value problems of multi-term fractional differential equations by using standard fixed point theorems. We demonstrate the application of the obtained results with the aid of examples. Some new results are also deduced by fixing the parameters involved in the problem at hand.
\end{abstract}

MSC: $34 \mathrm{~A} 08 ; 34 \mathrm{~B} 10$

Keywords: Caputo fractional derivative; Multi-term fractional differential equations; Multi-point and multi-strip boundary conditions; Existence; Fixed point

\section{Introduction}

Multi-term fractional differential equations involve more than one fractional order differential operators and appear in the mathematical models of many real world problems. Bagley-Torvik [1] and Basset equations [2] are important examples of this class of equations.

Fractional differential equations find useful applications in several disciplines of science and engineering such as blood flow phenomena, virology, bio-engineering, image processing, control theory, etc. For details and examples, see [3-7].

The literature on initial and boundary value problems of differential equations and inclusions containing a single fractional order operator is now much enriched and one can find useful results in a series of articles [8-19] and the references cited therein. However, the topic of boundary value problems of differential equations and inclusions containing more than two fractional order operators needs to be investigated. For some works on differential equations and inclusions involving two fractional order operators (sequential fractional differential equations) can be found in [20-23].

In this paper, we introduce and investigate a new boundary value problem of multi-term fractional differential equations supplemented with nonlocal multi-point and multi-strip boundary conditions given by

$$
\left(\delta_{2}{ }^{\mathrm{c}} D^{\alpha+2}+\delta_{1}{ }^{\mathrm{c}} D^{\alpha+1}+\delta_{0}{ }^{\mathrm{c}} D^{\alpha}\right) x(t)=f(t, x(t)), \quad 0<\alpha<1,0<t<1,
$$

(c) The Author(s) 2018. This article is distributed under the terms of the Creative Commons Attribution 4.0 International License (http://creativecommons.org/licenses/by/4.0/), which permits unrestricted use, distribution, and reproduction in any medium, provided you give appropriate credit to the original author(s) and the source, provide a link to the Creative Commons license, and indicate if changes were made. 


$$
x(0)=0, \quad x(\xi)=\sum_{i=1}^{n} j_{i} x\left(\eta_{i}\right), \quad x(1)=\sum_{i=1}^{k} \lambda_{i} \int_{v_{i}}^{\sigma_{i}} x(s) d s
$$

where ${ }^{\mathrm{c}} D^{\alpha}$ denotes the Caputo fractional derivative of order $\alpha, f:[0,1] \times \mathbb{R} \rightarrow \mathbb{R}$ is a given continuous function, $0<\xi<\eta_{1}<\eta_{2}<\cdots<\eta_{n}<v_{1}<\sigma_{1}<v_{2}<\sigma_{2}<\cdots<v_{k}<\sigma_{k}<1, j_{i} \in \mathbb{R}$, $i=1, \ldots, n, \lambda_{i} \in \mathbb{R}, i=1, \ldots, k, \delta_{i}$ are real numbers $\{i=0,1,2\}$, with $\delta_{2} \neq 0$.

The rest of the paper is organized as follows. In Sect. 2, we recall some preliminary ideas of fractional calculus and prove some important lemmas. Section 3 contains existence and uniqueness results for the problem (1.1)-(1.2) with $\delta_{1}^{2}-4 \delta_{0} \delta_{2}>0$, which are obtained by applying some well-known theorems of the fixed point theory. Though the tools of the fixed point theory are standard, their exposition helps to develop the existence theory for the given problem. In Sects. 4 and 5, we outline the idea for dealing with the problem (1.1)(1.2) involving the cases $\delta_{1}^{2}-4 \delta_{0} \delta_{2}=0$ and $\delta_{1}^{2}-4 \delta_{0} \delta_{2}<0$, respectively. The last section describes the importance and the scope of the obtained work.

\section{Basic results}

Before presenting some auxiliary results, let us recall some preliminary concepts of fractional calculus $[24,25]$.

Definition 2.1 Let $g$ be a locally integrable real-valued function on $-\infty \leq a<t<b \leq+\infty$. The Riemann-Liouville fractional integral $I_{a}^{q}$ of order $q \in \mathbb{R}(q>0)$ is defined as

$$
I_{a}^{q} g(t)=\left(g * K_{q}\right)(t)=\frac{1}{\Gamma(q)} \int_{a}^{t}(t-s)^{q-1} g(s) d s
$$

where $K_{q}(t)=\frac{t^{q-1}}{\Gamma(q)}, \Gamma$ denotes the Euler gamma function.

Definition 2.2 Let $g \in L^{1}[a, b],-\infty \leq a<t<b \leq+\infty$ and $g * K_{m-q} \in W^{m, 1}[a, b], m=$ $[q]+1, q>0$, where $W^{m, 1}[a, b]$ is the Sobolev space defined as

$$
W^{m, 1}[a, b]=\left\{g \in L^{1}[a, b]: \frac{d^{m}}{d t^{m}} g \in L^{1}[a, b]\right\} .
$$

The Riemann-Liouville fractional derivative $D_{a}^{q}$ of order $q>0(m-1<q<m, m \in \mathbb{N})$ is defined as

$$
D_{a}^{q} g(t)=\frac{d^{m}}{d t^{m}} I_{a}^{1-q} g(t)=\frac{1}{\Gamma(m-q)} \frac{d^{m}}{d t^{m}} \int_{a}^{t}(t-s)^{m-1-q} g(s) d s .
$$

Definition 2.3 Let $g \in L^{1}[a, b],-\infty \leq a<t<b \leq+\infty$ and $g * K_{m-q} \in W^{m, 1}[a, b], m=[q]$, $q>0$. The Caputo fractional derivative ${ }^{\mathrm{c}} D_{a}^{q}$ of order $q \in \mathbb{R}(m-1<q<m, m \in \mathbb{N})$ is defined as

$$
{ }^{\mathrm{c}} D_{a}^{q} g(t)=D_{a}^{q}\left[g(t)-g(a)-g^{\prime}(a) \frac{(t-a)}{1 !}-\cdots-g^{(m-1)}(a) \frac{(t-a)^{m-1}}{(m-1) !}\right] .
$$

If $g \in C^{m}[a, b]$, then the Caputo fractional derivative ${ }^{\mathrm{c}} D_{a}^{q}$ of order $q \in \mathbb{R}(m-1<q<m$, $m \in \mathbb{N}$ ) is defined as

$$
{ }^{\mathrm{c}} D_{a}^{q}[g](t)=I_{a}^{1-q} f^{(m)}(t)=\frac{1}{\Gamma(m-q)} \int_{a}^{t}(t-s)^{m-1-q} g^{(m)}(s) d s .
$$


In the sequel, the Riemann-Liouville fractional integral $I_{a}^{q}$ and the Caputo fractional derivative ${ }^{\mathrm{c}} D_{a}^{q}$ with $a=0$ are respectively denoted by $I^{q}$ and ${ }^{\mathrm{c}} D^{q}$.

Property 2.4 ([24]) With the given notations, the following equality holds:

$$
I^{q}\left({ }^{\mathrm{c}} D^{q} \varphi(t)\right)=\varphi(t)-c_{0}-c_{1} t-\cdots-c_{n-1} t^{n-1}, \quad t>0, n-1<q<n,
$$

where $c_{i}(i=1, \ldots, n-1)$ are arbitrary constants.

Definition 2.5 A function $x \in C^{3}[0,1]$ satisfying (1.1)-(1.2) is called a solution of this problem on $[0,1]$.

The following lemma associated with the linear variant of problem (1.1)-(1.2) plays an important role in the sequel.

Lemma 2.6 For any $y \in C([0,1], \mathbb{R})$ and $\delta_{1}^{2}-4 \delta_{0} \delta_{2}>0$, the solution of linear multi-term fractional differential equation

$$
\left(\delta_{2}{ }^{\mathrm{c}} D^{\alpha+2}+\delta_{1}{ }^{\mathrm{c}} D^{\alpha+1}+\delta_{0}{ }^{\mathrm{c}} D^{\alpha}\right) x(t)=y(t), \quad 0<\alpha<1,0<t<1,
$$

supplemented with the boundary conditions (1.2) is given by

$$
\begin{aligned}
x(t)= & \frac{1}{\hat{\delta}}\left\{\int_{0}^{t} \int_{0}^{s}\left(e^{m_{2}(t-s)}-e^{m_{1}(t-s)}\right) \frac{(s-u)^{\alpha-1}}{\Gamma(\alpha)} y(u) d u d s\right. \\
& +\rho_{1}(t)\left[\int_{0}^{\xi} \int_{0}^{s}\left(e^{m_{2}(\xi-s)}-e^{m_{1}(\xi-s)}\right) \frac{(s-u)^{\alpha-1}}{\Gamma(\alpha)} y(u) d u d s\right. \\
& \left.-\sum_{i=1}^{n} j_{i} \int_{0}^{\eta_{i}} \int_{0}^{s}\left(e^{m_{2}\left(\eta_{i}-s\right)}-e^{m_{1}\left(\eta_{i}-s\right)}\right) \frac{(s-u)^{\alpha-1}}{\Gamma(\alpha)} y(u) d u d s\right] \\
& +\rho_{2}(t)\left[\int_{0}^{1} \int_{0}^{s}\left(e^{m_{2}(1-s)}-e^{m_{1}(1-s)}\right) \frac{(s-u)^{\alpha-1}}{\Gamma(\alpha)} y(u) d u d s\right. \\
& \left.\left.-\sum_{i=1}^{k} \lambda_{i} \int_{v_{i}}^{\sigma_{i}} \int_{0}^{s}\left(\frac{\left(e^{m_{2}\left(\sigma_{i}-s\right)}-1\right)}{m_{2}}-\frac{\left(e^{m_{1}\left(\sigma_{i}-s\right)}-1\right)}{m_{1}}\right) \frac{(s-u)^{\alpha-1}}{\Gamma(\alpha)} y(u) d u d s\right]\right\},
\end{aligned}
$$

where

$$
\begin{aligned}
& m_{1}=\frac{-\delta_{1}-\sqrt{\delta_{1}^{2}-4 \delta_{0} \delta_{2}}}{2 \delta_{2}}, \quad m_{2}=\frac{-\delta_{1}+\sqrt{\delta_{1}^{2}-4 \delta_{0} \delta_{2}}}{2 \delta_{2}}, \\
& \delta_{2}\left(m_{2}-m_{1}\right)=\frac{\sqrt{\delta_{1}^{2}-4 \delta_{0} \delta_{2}}}{2}=\hat{\delta}, \\
& \rho_{1}(t)=\frac{\omega_{4} \varrho_{1}(t)-\omega_{3} \varrho_{2}(t)}{\mu_{1}}, \quad \rho_{2}(t)=\frac{\omega_{1} \varrho_{2}(t)-\omega_{2} \varrho_{1}(t)}{\mu_{1}}, \\
& \varrho_{1}(t)=\frac{\delta_{2}\left[m_{1}\left(1-e^{m_{2} t}\right)-m_{2}\left(1-e^{m_{1} t}\right)\right]}{\delta_{0}}, \\
& \varrho_{2}(t)=\hat{\delta}\left(e^{m_{2} t}-e^{m_{1} t}\right), \quad m_{1} m_{2}=\frac{\delta_{0}}{\delta_{2}}, \quad \mu_{1}=\omega_{1} \omega_{4}-\omega_{2} \omega_{3} \neq 0,
\end{aligned}
$$




$$
\begin{aligned}
\omega_{1}= & \frac{\delta_{2}}{\delta_{0}}\left[m_{2}\left(1-\sum_{i=1}^{n} j_{i}-e^{m_{1} \xi}+\sum_{i=1}^{n} j_{i} e^{m_{1} \eta_{i}}\right)\right. \\
& \left.-m_{1}\left(1-\sum_{i=1}^{n} j_{i}-e^{m_{2} \xi}+\sum_{i=1}^{n} j_{i} e^{m_{2} \eta_{i}}\right)\right], \\
\omega_{2}= & \hat{\delta}\left(e^{m_{1} \xi}-e^{m_{2} \xi}-\sum_{i=1}^{n} j_{i} e^{m_{1} \eta_{i}}+\sum_{i=1}^{n} j_{i} e^{m_{2} \eta_{i}}\right), \\
\omega_{3}= & \frac{\delta_{2}}{\delta_{0}}\left[m_{2}\left(1-e^{m_{1}}-\sum_{i=1}^{k} \lambda_{i}\left(\sigma_{i}-v_{i}\right)+\sum_{i=1}^{k} \lambda_{i} / m_{1}\left(e^{m_{1} \sigma_{i}}-e^{m_{1} v_{i}}\right)\right)\right. \\
& \left.-m_{1}\left(1-e^{m_{2}}-\sum_{i=1}^{k} \lambda_{i}\left(\sigma_{i}-v_{i}\right)+\sum_{i=1}^{k} \lambda_{i} / m_{2}\left(e^{m_{2} \sigma_{i}}-e^{m_{2} v_{i}}\right)\right)\right], \\
\omega_{4}= & \hat{\delta}\left(e^{m_{1}}-e^{m_{2}}-\sum_{i=1}^{k} \lambda_{i} / m_{1}\left(e^{m_{1} \sigma_{i}}-e^{m_{1} v_{i}}\right)+\sum_{i=1}^{k} \lambda_{i} / m_{2}\left(e^{m_{2} \sigma_{i}}-e^{m_{2} v_{i}}\right)\right) .
\end{aligned}
$$

Proof Applying the operator $I^{\alpha}$ on (2.2) and using (2.1), we get

$$
\left(\delta_{2} D^{2}+\delta_{1} D+\delta_{0}\right) x(t)=\int_{0}^{t} \frac{(t-s)^{\alpha-1}}{\Gamma(\alpha)} y(s) d s+c_{1},
$$

where $c_{1}$ is an arbitrary constant. By the method of variation of parameters, the solution of (2.5) can be written as

$$
\begin{aligned}
x(t)= & c_{2} e^{m_{1} t}+c_{3} e^{m_{2} t}-\frac{1}{\hat{\delta}}\left[\int_{0}^{t} e^{m_{1}(t-s)}\left(\int_{0}^{s} \frac{(s-u)^{\alpha-1}}{\Gamma(\alpha)} y(u) d u+c_{1}\right) d s\right. \\
& \left.+\int_{0}^{t} e^{m_{2}(t-s)}\left(\int_{0}^{s} \frac{(s-u)^{\alpha-1}}{\Gamma(\alpha)} y(u) d u+c_{1}\right) d s\right]
\end{aligned}
$$

where $m_{1}$ and $m_{2}$ are given by (2.4). Using $x(0)=0$ in (2.6) and simplifying the coefficient of $c_{1}$, we get

$$
\begin{aligned}
x(t)= & c_{1}\left(\frac{\delta_{2}\left[m_{2}\left(1-e^{m_{1} t}\right)-m_{1}\left(1-e^{m_{2} t}\right)\right]}{\delta_{0} \hat{\delta}}\right)+c_{2}\left(e^{m_{1} t}-e^{m_{2} t}\right) \\
& +\frac{1}{\hat{\delta}}\left[\int_{0}^{t}\left(e^{m_{2}(t-s)}-e^{m_{1}(t-s)}\right)\left(\int_{0}^{s} \frac{(s-u)^{\alpha-1}}{\Gamma(\alpha)} y(u) d u\right) d s\right],
\end{aligned}
$$

which, together with the conditions $x(\xi)=\sum_{i=1}^{n} j_{i} x\left(\eta_{i}\right)$ and $x(1)=\sum_{i=1}^{k} \lambda_{i} \int_{v_{i}}^{\sigma_{i}} x(s) d s$, yields the following system of equations in the unknown constants $c_{1}$ and $c_{2}$ :

$$
\begin{aligned}
& c_{1} \omega_{1}+c_{2} \omega_{2}=V_{1}, \\
& c_{1} \omega_{3}+c_{2} \omega_{4}=V_{2},
\end{aligned}
$$

where $\hat{\delta}$ and $\omega_{i}(i=1,2,3,4)$ are given by (2.4), and

$$
\begin{aligned}
V_{1}= & -\int_{0}^{\xi} \int_{0}^{s}\left(e^{m_{2}(\xi-s)}-e^{m_{1}(\xi-s)}\right) \frac{(s-u)^{\alpha-1}}{\Gamma(\alpha)} y(u) d u d s \\
& +\sum_{i=1}^{n} j_{i} \int_{0}^{\eta_{i}} \int_{0}^{s}\left(e^{m_{2}\left(\eta_{i}-s\right)}-e^{m_{1}\left(\eta_{i}-s\right)}\right) \frac{(s-u)^{\alpha-1}}{\Gamma(\alpha)} y(u) d u d s,
\end{aligned}
$$




$$
\begin{aligned}
V_{2}= & -\int_{0}^{1} \int_{0}^{s}\left(e^{m_{2}(1-s)}-e^{m_{1}(1-s)}\right) \frac{(s-u)^{\alpha-1}}{\Gamma(\alpha)} y(u) d u d s \\
& +\sum_{i=1}^{k} \lambda_{i} \int_{v_{i}}^{\sigma_{i}} \int_{0}^{s}\left[\frac{\left(e^{m_{1}(\sigma-s)}-1\right)}{m_{1}}-\frac{\left(e^{m_{2}(\sigma-s)}-1\right)}{m_{2}}\right] \frac{(s-u)^{\alpha-1}}{\Gamma(\alpha)} y(u) d u d s .
\end{aligned}
$$

Solving the system (2.8)-(2.9), we find that

$$
c_{1}=\frac{V_{1} \omega_{4}-V_{2} \omega_{2}}{\mu_{1}}, \quad c_{2}=\frac{V_{2} \omega_{1}-V_{1} \omega_{3}}{\mu_{1}} .
$$

Substituting the value of $c_{1}$ and $c_{2}$ in (2.7), we obtain the solution (2.3). This completes the proof.

Lemma 2.7 For any $y \in C([0,1], \mathbb{R})$ and $\delta_{1}^{2}-4 \delta_{0} \delta_{2}=0$, the solution of linear multi-term fractional differential equation

$$
\left(\delta_{2}{ }^{\mathrm{c}} D^{\alpha+2}+\delta_{1}{ }^{\mathrm{c}} D^{\alpha+1}+\delta_{0}{ }^{\mathrm{c}} D^{\alpha}\right) x(t)=y(t), \quad 0<\alpha<1,0<t<1,
$$

supplemented with the boundary conditions (1.2) is given by

$$
\begin{aligned}
x(t)= & \frac{1}{\delta_{2}}\left\{\int_{0}^{t} \int_{0}^{s}(t-s) e^{m(t-s)} \frac{(s-u)^{\alpha-1}}{\Gamma(\alpha)} y(u) d u d s\right. \\
& +\chi_{1}(t)\left[\int_{0}^{\xi} \int_{0}^{s}(\xi-s) e^{m(\xi-s)} \frac{(s-u)^{\alpha-1}}{\Gamma(\alpha)} y(u) d u d s\right. \\
& \left.-\sum_{i=1}^{n} j_{i} \int_{0}^{\eta_{i}} \int_{0}^{s}\left(\eta_{i}-s\right) e^{m\left(\eta_{i}-s\right)} \frac{(s-u)^{\alpha-1}}{\Gamma(\alpha)} y(u) d u d s\right] \\
& +\chi_{2}(t)\left[\int_{0}^{1} \int_{0}^{s}(1-s) e^{m(1-s)} \frac{(s-u)^{\alpha-1}}{\Gamma(q)} y(u) d u d s\right. \\
& \left.\left.-\sum_{i=1}^{k} \lambda_{i} \int_{v_{i}}^{\sigma_{i}} \int_{0}^{s}\left(\frac{m\left(\sigma_{i}-s\right) e^{m\left(\sigma_{i}-s\right)}-e^{m\left(\sigma_{i}-s\right)}+1}{m^{2}}\right) \frac{(s-u)^{q-1}}{\Gamma(q)} y(u) d u d s\right]\right\}
\end{aligned}
$$

where

$$
\begin{aligned}
& m=\frac{-\delta_{1}}{2 \delta_{2}}, \quad \chi_{1}(t)=\frac{\varpi_{3} z_{2}(t)-\varpi_{4} z_{1}(t)}{\mu_{2}}, \quad \chi_{2}(t)=\frac{\varpi_{2} z_{1}(t)-\varpi_{1} z_{2}(t)}{\mu_{2}}, \\
& z_{1}(t)=\frac{m t e^{m t}-e^{m t}+1}{m^{2}}, \quad z_{2}(t)=\delta_{2} t e^{m t} \\
& \varpi_{1}=\frac{m \xi e^{m \xi}-e^{m \xi}+1-\sum_{i=1}^{n} j_{i}\left(m \eta_{i} e^{m \eta_{i}}-e^{m \eta_{i}}+1\right)}{m^{2}} \\
& \varpi_{2}=\delta_{2}\left(\xi e^{m \xi}-\sum_{i=1}^{n} j_{i} \eta_{i} e^{m \eta_{i}}\right) \\
& \varpi_{3}=\frac{1}{m^{3}}\left[m^{2} e^{m}-m e^{m}+m-m \sum_{i=1}^{k} \lambda_{i}\left(\sigma_{i} e^{m \sigma_{i}}-v_{i} e^{m v_{i}}\right)\right.
\end{aligned}
$$




$$
\begin{aligned}
&\left.+2 \sum_{i=1}^{k} \lambda_{i}\left(e^{m \sigma_{i}}-e^{m v_{i}}\right)-m \sum_{i=1}^{k} \lambda_{i}\left(\sigma_{i}-v_{i}\right)\right] \\
& \varpi_{4}= \delta_{2}\left(\frac{m^{2} e^{m}-m \sum_{i=1}^{k} \lambda_{i}\left(\sigma_{i} e^{m \sigma_{i}}-v_{i} e^{m v_{i}}\right)+\sum_{i=1}^{k} \lambda_{i}\left(e^{m \sigma_{i}}-e^{m v_{i}}\right)}{m^{2}}\right), \\
& \mu_{2}=\varpi_{1} \varpi_{4}-\varpi_{2} \varpi_{3} \neq 0 .
\end{aligned}
$$

Proof Since the proof is similar to that of Lemma 2.6, we omit it.

Lemma 2.8 For any $y \in C([0,1], \mathbb{R})$ and $\delta_{1}^{2}-4 \delta_{0} \delta_{2}<0$, the solution of linear multi-term fractional differential equation

$$
\left(\delta_{2}{ }^{\mathrm{c}} D^{\alpha+2}+\delta_{1}{ }^{\mathrm{c}} D^{\alpha+1}+\delta_{0}{ }^{\mathrm{c}} D^{\alpha}\right) x(t)=y(t), \quad 0<\alpha<1,0<t<1,
$$

supplemented with the boundary conditions (1.2) is given by

$$
\begin{aligned}
x(t)= & \frac{1}{\delta_{2} b}\left\{\int_{0}^{t} \int_{0}^{s} e^{-a(t-s)} \sin b(t-s) \frac{(s-u)^{\alpha-1}}{\Gamma(\alpha)} y(u) d u d s\right. \\
& +\tau_{1}(t)\left[\int_{0}^{\xi} \int_{0}^{s} e^{-a(\xi-s)} \sin b(\xi-s) \frac{(s-u)^{\alpha-1}}{\Gamma(\alpha)} y(u) d u d s\right. \\
& \left.-\sum_{i=1}^{n} j_{i} \int_{0}^{\eta_{i}} \int_{0}^{s} e^{-a\left(\eta_{i}-s\right)} \sin b\left(\eta_{i}-s\right) \frac{(s-u)^{\alpha-1}}{\Gamma(\alpha)} y(u) d u d s\right] \\
& +\tau_{2}(t)\left[\int_{0}^{1} \int_{0}^{s} e^{-a(1-s)} \sin b(1-s) \frac{(s-u)^{q-1}}{\Gamma(q)} y(u) d u d s\right. \\
& -\frac{\sum_{i=1}^{k} \lambda_{i}}{a^{2}+b^{2}} \int_{v_{i}}^{\sigma_{i}} \int_{0}^{s}\left(b-b e^{-a\left(\sigma_{i}-s\right)} \cos b\left(\sigma_{i}-s\right)\right. \\
& \left.\left.\left.-a e^{-a\left(\sigma_{i}-s\right)} \sin b\left(\sigma_{i}-s\right)\right) \frac{(s-u)^{\alpha-1}}{\Gamma(\alpha)} y(u) d u d s\right]\right\}
\end{aligned}
$$

where

$$
\begin{aligned}
& m_{1,2}=-a \pm b i, \quad a=\frac{\delta_{1}}{2 \delta_{2}}, \quad b=\frac{\sqrt{4 \delta_{0} \delta_{2}-\delta_{1}^{2}}}{2 \delta_{2}}, \\
& \tau_{1}(t)=\frac{q_{3} \nu_{2}(t)-q_{4} \nu_{1}(t)}{\mu_{3}}, \quad \tau_{2}(t)=\frac{q_{2} v_{1}(t)-q_{1} \nu_{2}(t)}{\mu_{3}}, \\
& v_{1}(t)=\frac{b+b e^{-a t} \cos b t-a e^{-a t} \sin b t}{a^{2}+b^{2}}, \quad v_{2}(t)=\delta_{2} b e^{-a t} \sin b t \\
& q_{1}=\frac{b-b e^{-a \xi} \cos b \xi-a e^{-a \xi} \sin b \xi-\sum_{i=1}^{n} j_{i}\left(b-b e^{-a \eta_{i}} \cos b \eta_{i}-a e^{-a \eta_{i}} \sin b \eta_{i}\right)}{a^{2}+b^{2}}, \\
& q_{2}=\delta_{2} b\left(e^{-a \xi} \sin b \xi-\sum_{i=1}^{n} j_{i} e^{-a \eta_{i}} \sin b \eta_{i}\right), \\
& q_{3}=\frac{1}{a^{2}+b^{2}}\left[b-b e^{-a} \cos b-a e^{-a} \sin b-b \sum_{i=1}^{k} \lambda_{i}\left(\sigma_{i}-v_{i}\right)\right.
\end{aligned}
$$




$$
\begin{aligned}
& -\frac{a^{2}-b^{2}}{a^{2}+b^{2}} \sum_{i=1}^{k} \lambda_{i}\left(e^{-a \sigma_{i}} \sin b \sigma_{i}-e^{-a v_{i}} \sin b v_{i}\right) \\
& \left.-\frac{2 a b}{a^{2}+b^{2}} \sum_{i=1}^{k} \lambda_{i}\left(e^{-a \sigma_{i}} \cos b \sigma_{i}-e^{-a v_{i}} \cos b v_{i}\right)\right], \\
q_{4}= & \delta_{2} b\left[e^{-a} \sin b+\frac{\sum_{i=1}^{k} \lambda_{i}}{a^{2}+b^{2}}\left(b e^{-a \sigma_{i}} \cos b \sigma_{i}-b e^{-a v_{i}} \cos b v_{i}\right.\right. \\
& \left.\left.+a e^{-a \sigma_{i}} \sin b \sigma_{i}-a e^{-a v_{i}} \sin b v_{i}\right)\right], \\
\mu_{3}= & q_{1} q_{4}-q_{2} q_{3} \neq 0 .
\end{aligned}
$$

Proof We do not provide the proof as it is similar to that of Lemma 2.6.

\section{Existence and uniqueness results for the case $\delta_{1}{ }^{2}-4 \delta_{0} \delta_{2}>0$}

Denote by $\mathcal{C}=C([0,1], \mathbb{R})$ the Banach space of all continuous functions from $[0,1]$ to $\mathbb{R}$ endowed with the norm defined by $\|x\|=\sup \{|x(t)|: t \in[0,1]\}$.

By Lemma 2.6, we transform the problem (1.1)-(1.2) with $\delta_{1}{ }^{2}-4 \delta_{0} \delta_{2}>0$ into a fixed point problem as

$$
x=\mathcal{J} x,
$$

where the operator $\mathcal{J}: \mathcal{C} \rightarrow \mathcal{C}$ is defined by

$$
\begin{aligned}
(\mathcal{J} x)(t)= & \frac{1}{\delta}\left\{\int_{0}^{t} \int_{0}^{s}\left(e^{m_{2}(t-s)}-e^{m_{1}(t-s)}\right) \frac{(s-u)^{\alpha-1}}{\Gamma(\alpha)} f(u, x(u)) d u d s\right. \\
& +\rho_{1}(t)\left[\int_{0}^{\xi} \int_{0}^{s}\left(e^{m_{2}(\xi-s)}-e^{m_{1}(\xi-s)}\right) \frac{(s-u)^{\alpha-1}}{\Gamma(\alpha)} f(u, x(u)) d u d s\right. \\
& \left.-\sum_{i=1}^{n} j_{i} \int_{0}^{\eta_{i}} \int_{0}^{s}\left(e^{m_{2}\left(\eta_{i}-s\right)}-e^{m_{1}\left(\eta_{i}-s\right)}\right) \frac{(s-u)^{\alpha-1}}{\Gamma(\alpha)} f(u, x(u)) d u d s\right] \\
& +\rho_{2}(t)\left[\int_{0}^{1} \int_{0}^{s}\left(e^{m_{2}(1-s)}-e^{m_{1}(1-s)}\right) \frac{(s-u)^{\alpha-1}}{\Gamma(\alpha)} f(u, x(u)) d u d s\right. \\
& -\sum_{i=1}^{k} \lambda_{i} \int_{v_{i}}^{\sigma_{i}} \int_{0}^{s}\left(\frac{\left(e^{m_{2}(\sigma-s)}-1\right)}{m_{2}}-\frac{\left(e^{m_{1}(\sigma-s)}-1\right)}{m_{1}}\right) \\
& \left.\left.\times \frac{(s-u)^{\alpha-1}}{\Gamma(\alpha)} f(u, x(u)) d u d s\right]\right\},
\end{aligned}
$$

with $\rho_{1}(t)$ and $\rho_{2}(t)$ given by (2.4).

In the sequel, for the sake of computational convenience, we set

$$
\begin{aligned}
& \widehat{\rho}_{1}=\max _{t \in[0,1]}\left|\rho_{1}(t)\right|, \quad \widehat{\rho}_{2}=\max _{t \in[0,1]}\left|\rho_{2}(t)\right|, \\
& \varepsilon=\max _{t \in[0,1]}\left|m_{2}\left(1-e^{m_{1} t}\right)-m_{1}\left(1-e^{m_{2} t}\right)\right|,
\end{aligned}
$$




$$
\begin{aligned}
\phi= & \frac{\left|\delta_{2}\right|}{\left|\delta_{0} \hat{\delta}\right| \Gamma(\alpha+1)}\left\{\varepsilon+\widehat{\rho}_{1}\left[\xi^{\alpha}\left|m_{2}\left(1-e^{m_{1} \xi}\right)-m_{1}\left(1-e^{m_{2} \xi}\right)\right|\right.\right. \\
& \left.+\sum_{i=1}^{n}\left|j_{i}\right| \eta_{i}^{\alpha}\left|m_{2}\left(1-e^{m_{1} \eta_{i}}\right)-m_{1}\left(1-e^{m_{2} \eta_{i}}\right)\right|\right] \\
& +\widehat{\rho}_{2}\left[\left|m_{2}\left(1-e^{m_{1}}\right)-m_{1}\left(1-e^{m_{2}}\right)\right|\right. \\
& +\frac{\left|\delta_{2}\right|}{\left|\delta_{0}\right|} \sum_{i=1}^{k}\left|\lambda_{i}\right| \sigma_{i}^{\alpha} \mid m_{2}^{2}\left(m_{1}\left(\sigma_{i}-v_{i}\right)-e^{m_{1}\left(\sigma_{i}-v_{i}\right)}+1\right) \\
& \left.\left.-m_{1}^{2}\left(m_{2}\left(\sigma_{i}-v_{i}\right)-e^{m_{2}\left(\sigma_{i}-v_{i}\right)}+1\right) \mid\right]\right\} \\
\phi_{1}= & \phi-\frac{\left|\delta_{2}\right| \varepsilon}{\left|\delta_{0} \hat{\delta}\right| \Gamma(\alpha+1)} .
\end{aligned}
$$

Now the stage is set to present our main results. In the first result, we use Krasnoselskii's fixed point theorem to prove the existence of solutions for the problem (1.1)-(1.2) with $\delta_{1}^{2}-4 \delta_{0} \delta_{2}>0$.

Theorem 3.1 (Krasnoselskii's fixed point theorem [26]) Let $Y$ be a bounded, closed, convex, and nonempty subset of a Banach space $X$. Let $F_{1}$ and $F_{2}$ be operators satisfying the conditions: (i) $F_{1} y_{1}+F_{2} y_{2} \in Y$ whenever $y_{1}, y_{2} \in Y$; (ii) $F_{1}$ is compact and continuous; (iii) $F_{2}$ is a contraction mapping. Then there exists a $y \in Y$ such that $y=F_{1} y+F_{2} y$.

In the forthcoming analysis, we need the following assumptions:

(A $\left.\mathrm{A}_{1}\right)|f(t, x)-f(t, y)| \leq \ell\|x-y\|$, for all $t \in[0,1], x, y \in \mathbb{R}, \ell>0$.

$\left(\mathrm{A}_{2}\right)|f(t, x)| \leq \vartheta(t)$, for all $(t, x) \in[0,1] \times \mathbb{R}$ and $\vartheta \in C\left([0,1], \mathbb{R}^{+}\right)$.

Theorem 3.2 Let $f:[0,1] \times \mathbb{R} \rightarrow \mathbb{R}$ be a continuous function satisfying conditions $\left(\mathrm{A}_{1}\right)$ and $\left(\mathrm{A}_{2}\right)$. Then the problem (1.1)-(1.2), with $\delta_{1}^{2}-4 \delta_{0} \delta_{2}>0$, has at least one solution on $[0,1]$ if

$$
\ell \phi_{1}<1
$$

where $\phi_{1}$ is given by (3.2).

Proof Setting $\sup _{t \in[0,1]}|\vartheta(t)|=\|\vartheta\|$, we can fix

$$
\begin{aligned}
r \geq & \frac{\left|\delta_{2}\right||\vartheta| \mid}{\left|\delta_{0} \hat{\delta}\right| \Gamma(\alpha+1)}\left\{\varepsilon+\widehat{\rho}_{1}\left[\xi^{\alpha}\left|m_{2}\left(1-e^{m_{1} \xi}\right)-m_{1}\left(1-e^{m_{2} \xi}\right)\right|\right.\right. \\
& \left.+\sum_{i=1}^{n}\left|j_{i}\right| \eta_{i}^{\alpha}\left|m_{2}\left(1-e^{m_{1} \eta_{i}}\right)-m_{1}\left(1-e^{m_{2} \eta_{i}}\right)\right|\right]+\widehat{\rho}_{2}\left[\left|m_{2}\left(1-e^{m_{1}}\right)-m_{1}\left(1-e^{m_{2}}\right)\right|\right. \\
& \left.\left.+\frac{\delta_{2}}{\delta_{0}} \sum_{i=1}^{k}\left|\lambda_{i}\right|\left|m_{2}^{2}\left(m_{1}\left(\sigma_{i}-v_{i}\right)-e^{m_{1}\left(\sigma_{i}-v_{i}\right)}+1\right)-m_{1}^{2}\left(m_{2}\left(\sigma_{i}-v_{i}\right)-e^{m_{2}\left(\sigma_{i}-v_{i}\right)}+1\right)\right|\right]\right\}
\end{aligned}
$$


and define $B_{r}=\{x \in \mathcal{C}:\|x\| \leq r\}$. Introduce the operators $\mathcal{J}_{1}$ and $\mathcal{J}_{2}$ on $B_{r}$ as follows:

$$
\left(\mathcal{J}_{1} x\right)(t)=\frac{1}{\hat{\delta}} \int_{0}^{t} \int_{0}^{s}\left(e^{m_{2}(t-s)}-e^{m_{1}(t-s)}\right) \frac{(s-u)^{\alpha-1}}{\Gamma(\alpha)} f(u, x(u)) d u d s
$$

and

$$
\begin{aligned}
\left(\mathcal{J}_{2} x\right)(t)= & \frac{1}{\hat{\delta}}\left\{\rho _ { 1 } ( t ) \left[\int_{0}^{\xi} \int_{0}^{s}\left(e^{m_{2}(\xi-s)}-e^{m_{1}(\xi-s)}\right) \frac{(s-u)^{\alpha-1}}{\Gamma(\alpha)} f(u, x(u)) d u d s\right.\right. \\
& \left.-\sum_{i=1}^{n} j_{i} \int_{0}^{\eta_{i}} \int_{0}^{s}\left(e^{m_{2}\left(\eta_{i}-s\right)}-e^{m_{1}\left(\eta_{i}-s\right)}\right) \frac{(s-u)^{\alpha-1}}{\Gamma(\alpha)} f(u, x(u)) d u d s\right] \\
& +\rho_{2}(t)\left[\int_{0}^{1} \int_{0}^{s}\left(e^{m_{2}(1-s)}-e^{m_{1}(1-s)}\right) \frac{(s-u)^{\alpha-1}}{\Gamma(\alpha)} f(u, x(u)) d u d s\right. \\
& -\sum_{i=1}^{k} \lambda_{i} \int_{v_{i}}^{\sigma_{i}} \int_{0}^{s}\left(\frac{\left(e^{m_{2}\left(\sigma_{i}-s\right)}-1\right)}{m_{2}}-\frac{\left(e^{m_{1}\left(\sigma_{i}-s\right)}-1\right)}{m_{1}}\right) \\
& \left.\left.\times \frac{(s-u)^{\alpha-1}}{\Gamma(\alpha)} f(u, x(u)) d u d s\right]\right\}
\end{aligned}
$$

Observe that $\mathcal{J}=\mathcal{J}_{1}+\mathcal{J}_{2}$. For $x, y \in B_{r}$, we have

$$
\begin{aligned}
& \left\|\mathcal{J}_{1} x+\mathcal{J}_{2} y\right\| \\
& =\sup _{t \in[0,1]}\left|\left(\mathcal{J}_{1} x\right)(t)+\left(\mathcal{J}_{2} y\right)(t)\right| \\
& \leq \frac{1}{|\hat{\delta}|} \sup _{t \in[0,1]}\left\{\int_{0}^{t} \int_{0}^{s}\left(e^{m_{2}(t-s)}-e^{m_{1}(t-s)}\right) \frac{(s-u)^{\alpha-1}}{\Gamma(\alpha)}|f(u, x(u))| d u d s\right. \\
& +\left|\rho_{1}(t)\right|\left[\int_{0}^{\xi} \int_{0}^{s}\left(e^{m_{2}(\xi-s)}-e^{m_{1}(\xi-s)}\right) \frac{(s-u)^{\alpha-1}}{\Gamma(\alpha)}|f(u, y(u))| d u d s\right. \\
& \left.+\sum_{i=1}^{n}\left|j_{i}\right| \int_{0}^{\eta_{i}} \int_{0}^{s}\left(e^{m_{2}\left(\eta_{i}-s\right)}-e^{m_{1}\left(\eta_{i}-s\right)}\right) \frac{(s-u)^{\alpha-1}}{\Gamma(\alpha)}|f(u, y(u))| d u d s\right] \\
& +\left|\rho_{2}(t)\right|\left[\int_{0}^{1} \int_{0}^{s}\left(e^{m_{2}(1-s)}-e^{m_{1}(1-s)}\right) \frac{(s-u)^{\alpha-1}}{\Gamma(\alpha)}|f(u, y(u))| d u d s\right. \\
& \left.\left.+\sum_{i=1}^{k}\left|\lambda_{i}\right| \int_{v_{i}}^{\sigma_{i}} \int_{0}^{s}\left(\frac{\left(e^{m_{2}\left(\sigma_{i}-s\right)}-1\right)}{m_{2}}-\frac{\left(e^{m_{1}\left(\sigma_{i}-s\right)}-1\right)}{m_{1}}\right) \frac{(s-u)^{\alpha-1}}{\Gamma(\alpha)}|f(u, y(u))| d u d s\right]\right\} \\
& \leq \frac{\|\vartheta\|}{|\hat{\delta}| \Gamma(\alpha+1)} \sup _{t \in[0,1]}\left\{t^{\alpha} \int_{0}^{t}\left|e^{m_{2}(t-s)}-e^{m_{1}(t-s)}\right| d s\right. \\
& +\left|\rho_{1}(t)\right|\left[\xi^{\alpha} \int_{0}^{\xi}\left|e^{m_{2}(\xi-s)}-e^{m_{1}(\xi-s)}\right| d s+\sum_{i=1}^{n}\left|j_{i}\right| \eta_{i}^{\alpha} \int_{0}^{\eta_{i}}\left|e^{m_{2}\left(\eta_{i}-s\right)}-e^{m_{1}\left(\eta_{i}-s\right)}\right| d s\right] \\
& +\left|\rho_{2}(t)\right|\left[\int_{0}^{1}\left|e^{m_{2}(1-s)}-e^{m_{1}(1-s)}\right| d s\right.
\end{aligned}
$$




$$
\begin{aligned}
& \left.\left.+\sum_{i=1}^{k}\left|\lambda_{i}\right| \sigma_{i}^{\alpha} \int_{v_{i}}^{\sigma_{i}}\left|\frac{\left(e^{m_{2}\left(\sigma_{i}-s\right)}-1\right)}{m_{2}}-\frac{\left(e^{m_{1}\left(\sigma_{i}-s\right)}-1\right)}{m_{1}}\right| d s\right]\right\} \\
\leq & \frac{\left|\delta_{2}\right|\|\vartheta\|}{\left|\delta_{0} \hat{\delta}\right| \Gamma(\alpha+1)}\left\{\varepsilon+\widehat{\rho}_{1}\left[\xi^{\alpha}\left|m_{2}\left(1-e^{m_{1} \xi}\right)-m_{1}\left(1-e^{m_{2} \xi}\right)\right|\right.\right. \\
& \left.+\sum_{i=1}^{n}\left|j_{i}\right| \eta_{i}^{\alpha}\left|m_{2}\left(1-e^{m_{1} \eta_{i}}\right)-m_{1}\left(1-e^{m_{2} \eta_{i}}\right)\right|\right]+\widehat{\rho}_{2}\left[\left|m_{2}\left(1-e^{m_{1}}\right)-m_{1}\left(1-e^{m_{2}}\right)\right|\right. \\
& +\frac{\left|\delta_{2}\right|}{\left|\delta_{0}\right|} \sum_{i=1}^{k}\left|\lambda_{i}\right| \sigma_{i}^{\alpha} \mid m_{2}^{2}\left(m_{1}\left(\sigma_{i}-v_{i}\right)-e^{m_{1}\left(\sigma_{i}-v_{i}\right)}+1\right) \\
& \left.\left.-m_{1}^{2}\left(m_{2}\left(\sigma_{i}-v_{i}\right)-e^{m_{2}\left(\sigma_{i}-v_{i}\right)}+1\right) \mid\right]\right\} \\
\leq & r .
\end{aligned}
$$

Thus $\mathcal{J}_{1} x+\mathcal{J}_{2} y \in B_{r}$. Using assumption $\left(\mathrm{A}_{1}\right)$ together with (3.3), we show that $\mathcal{J}_{2}$ is a contraction as follows:

$$
\begin{aligned}
& \left\|\mathcal{J}_{2} x-\mathcal{J}_{2} y\right\| \\
& =\sup _{t \in[0,1]}\left|\left(\mathcal{J}_{2} x\right)(t)-\left(\mathcal{J}_{2} y\right)(t)\right| \\
& \leq \frac{1}{|\hat{\delta}|} \sup _{t \in[0,1]}\left\{| \rho _ { 1 } ( t ) | \left[\int_{0}^{\xi} \int_{0}^{s}\left(e^{m_{2}(\xi-s)}-e^{m_{1}(\xi-s)}\right) \frac{(s-u)^{\alpha-1}}{\Gamma(\alpha)}\right.\right. \\
& \times|f(u, x(u))-f(u, y(u))| d u d s \\
& \left.+\sum_{i=1}^{n}\left|j_{i}\right| \int_{0}^{\eta_{i}} \int_{0}^{s}\left(e^{m_{2}\left(\eta_{i}-s\right)}-e^{m_{1}\left(\eta_{i}-s\right)}\right) \frac{(s-u)^{\alpha-1}}{\Gamma(\alpha)}|f(u, x(u))-f(u, y(u))| d u d s\right] \\
& +\left|\rho_{2}(t)\right|\left[\int_{0}^{1} \int_{0}^{s}\left(e^{m_{2}(1-s)}-e^{m_{1}(1-s)}\right) \frac{(s-u)^{\alpha-1}}{\Gamma(\alpha)}|f(u, x(u))-f(u, y(u))| d u d s\right. \\
& +\sum_{i=1}^{k}\left|\lambda_{i}\right| \int_{v_{i}}^{\sigma_{i}} \int_{0}^{s}\left(\frac{\left(e^{m_{2}\left(\sigma_{i}-s\right)}-1\right)}{m_{2}}-\frac{\left(e^{m_{1}\left(\sigma_{i}-s\right)}-1\right)}{m_{1}}\right) \\
& \left.\left.\times \frac{(s-u)^{\alpha-1}}{\Gamma(\alpha)}|f(u, x(u))-f(u, y(u))| d u d s\right]\right\} \\
& \leq \frac{\ell}{|\hat{\delta}|} \sup _{t \in[0,1]}\left\{| \rho _ { 1 } ( t ) | \left[\xi^{\alpha} \int_{0}^{\xi}\left|e^{m_{2}(\xi-s)}-e^{m_{1}(\xi-s)}\right| d s\right.\right. \\
& \left.+\sum_{i=1}^{n}\left|j_{i}\right| \eta_{i}^{\alpha} \int_{0}^{\eta_{i}}\left|e^{m_{2}\left(\eta_{i}-s\right)}-e^{m_{1}\left(\eta_{i}-s\right)}\right| d s\right] \\
& +\left|\rho_{2}(t)\right|\left[\int_{0}^{1} \int_{0}^{s}\left|e^{m_{2}(1-s)}-e^{m_{1}(1-s)}\right| d u d s\right. \\
& \left.\left.+\sum_{i=1}^{k}\left|\lambda_{i}\right| \sigma_{i}^{\alpha} \int_{v_{i}}^{\sigma_{i}}\left|\frac{\left(e^{m_{2}\left(\sigma_{i}-s\right)}-1\right)}{m_{2}}-\frac{\left(e^{m_{1}\left(\sigma_{i}-s\right)}-1\right)}{m_{1}}\right| d s\right]\right\}\|x-y\|
\end{aligned}
$$




$$
\begin{aligned}
\leq & \frac{\left|\delta_{2}\right| \ell}{\left|\delta_{0} \hat{\delta}\right| \Gamma(\alpha+1)}\left\{\widehat { \rho } _ { 1 } \left[\xi^{\alpha}\left|m_{2}\left(1-e^{m_{1} \xi}\right)-m_{1}\left(1-e^{m_{2} \xi}\right)\right|\right.\right. \\
& \left.+\sum_{i=1}^{n}\left|j_{i}\right| \eta_{i}^{\alpha}\left|m_{2}\left(1-e^{m_{1} \eta_{i}}\right)-m_{1}\left(1-e^{m_{2} \eta_{i}}\right)\right|\right]+\widehat{\rho}_{2}\left[\left|m_{2}\left(1-e^{m_{1}}\right)-m_{1}\left(1-e^{m_{2}}\right)\right|\right. \\
& +\frac{\left|\delta_{2}\right|}{\left|\delta_{0}\right|} \sum_{i=1}^{k}\left|\lambda_{i}\right| \sigma_{i}^{\alpha} \mid m_{2}^{2}\left(m_{1}\left(\sigma_{i}-v_{i}\right)-e^{m_{1}\left(\sigma_{i}-v_{i}\right)}+1\right) \\
& \left.\left.-m_{1}^{2}\left(m_{2}\left(\sigma_{i}-v_{i}\right)-e^{m_{2}\left(\sigma_{i}-v_{i}\right)}+1\right) \mid\right]\right\}\|x-y\| \\
= & \ell \phi_{1}\|x-y\| .
\end{aligned}
$$

Note that continuity of $f$ implies that operator $\mathcal{J}_{1}$ is continuous. Also, $\mathcal{J}_{1}$ is uniformly bounded on $B_{r}$ as

$$
\left\|\mathcal{J}_{1} x\right\|=\sup _{t \in[0,1]}\left|\left(\mathcal{J}_{1} x\right)(t)\right| \leq \frac{\left|\delta_{2}\right|\|\vartheta\| \varepsilon}{\left|\delta_{0} \hat{\delta}\right| \Gamma(\alpha+1)} .
$$

Now we prove the compactness of operator $\mathcal{J}_{1}$. We define $\sup _{(t, x) \in[0,1] \times B_{r}}|f(t, x)|=\bar{f}$. Thus, for $0<t_{1}<t_{2}<1$, we have

$$
\begin{aligned}
& \left|\left(\mathcal{J}_{1} x\right)\left(t_{2}\right)-\left(\mathcal{J}_{1} x\right)\left(t_{1}\right)\right| \\
& =\frac{1}{|\hat{\delta}|} \mid \int_{0}^{t_{1}} \int_{0}^{s}\left[\left(e^{m_{2}\left(t_{2}-s\right)}-e^{m_{1}\left(t_{2}-s\right)}\right)-\left(e^{m_{2}\left(t_{1}-s\right)}-e^{m_{1}\left(t_{1}-s\right)}\right)\right] \\
& \quad \times \frac{(s-u)^{\alpha-1}}{\Gamma(\alpha)} f(u, x(u)) d u d s \\
& \quad+\int_{t_{1}}^{t_{2}} \int_{0}^{s}\left(e^{m_{2}\left(t_{2}-s\right)}-e^{m_{1}\left(t_{2}-s\right)}\right) \frac{(s-u)^{\alpha-1}}{\Gamma(\alpha)} f(u, x(u)) d u d s \mid \\
& \leq \frac{\left|\delta_{2}\right| \bar{f}}{\left|\delta_{0} \hat{\delta}\right| \Gamma(\alpha+1)}\left\{\left(t_{1}^{\alpha}-t_{2}^{\alpha}\right)\left|m_{1}\left(1-e^{m_{2}\left(t_{2}-t_{1}\right)}\right)-m_{2}\left(1-e^{m_{1}\left(t_{2}-t_{1}\right)}\right)\right|\right. \\
& \left.\quad+t_{1}^{\alpha}\left|m_{1}\left(e^{m_{2} t_{2}}-e^{m_{2} t_{1}}\right)-m_{2}\left(e^{m_{1} t_{2}}-e^{m_{1} t_{1}}\right)\right|\right\} \rightarrow 0, \quad \text { as } t_{1} \rightarrow t_{2},
\end{aligned}
$$

independent of $x \in B_{r}$. Thus, $\mathcal{J}_{1}$ is relatively compact on $B_{r}$. Hence, by the Arzelá-Ascoli Theorem, $\mathcal{J}_{1}$ is compact on $B_{r}$. Thus all the assumptions of Theorem 3.1 are satisfied. So, by the conclusion of Theorem 3.1, the problem (1.1)-(1.2) with $\delta_{1}{ }^{2}-4 \delta_{0} \delta_{2}>0$ has at least one solution on $[0,1]$. The proof is completed.

Remark 3.3 In the above theorem we can interchange the roles of operators $\mathcal{J}_{1}$ and $\mathcal{J}_{2}$ to obtain a second result by replacing (3.3) with the following condition:

$$
\frac{\left|\delta_{2}\right| \ell \varepsilon}{\left|\delta_{0} \hat{\delta}\right| \Gamma(\alpha+1)}<1
$$

In the next result, we prove the uniqueness of solutions for the problem (1.1)-(1.2) with $\delta_{1}^{2}-4 \delta_{0} \delta_{2}>0$ by applying Banach contraction mapping principle. 
Theorem 3.4 Assume that $f:[0,1] \times \mathbb{R} \rightarrow \mathbb{R}$ is a continuous function such that $\left(\mathrm{A}_{1}\right)$ is satisfied. Then there exists a unique solution for the problem (1.1)-(1.2), with $\delta_{1}{ }^{2}-4 \delta_{0} \delta_{2}>$ 0 , on $[0,1]$ if $\ell<1 / \phi$, where $\phi$ is given by $(3.2)$.

Proof Let us define $\sup _{t \in[0,1]}|f(t, 0)|=M$ and select $\bar{r} \geq \frac{\phi M}{1-\ell \phi}$ to show that $\mathcal{J} B_{\bar{r}} \subset B_{\bar{r}}$, where $B_{\bar{r}}=\{x \in \mathcal{C}:\|x\| \leq \bar{r}\}$ and $\mathcal{J}$ is defined by (3.1). Using condition $\left(\mathrm{A}_{1}\right)$, we have

$$
\begin{aligned}
|f(t, x)| & =|f(t, x)-f(t, 0)+f(t, 0)| \leq|f(t, x)-f(t, 0)|+|f(t, 0)| \\
& \leq \ell\|x\|+M \leq \ell \bar{r}+M .
\end{aligned}
$$

Then, for $x \in B_{\bar{r}}$, we obtain

$$
\begin{aligned}
& \|\mathcal{J}(x)\| \\
& =\sup _{t \in[0,1]}|\mathcal{J}(x)(t)| \\
& \leq \frac{1}{|\hat{\delta}|} \sup _{t \in[0,1]}\left\{\int_{0}^{t} \int_{0}^{s}\left(e^{m_{2}(t-s)}-e^{m_{1}(t-s)}\right) \frac{(s-u)^{\alpha-1}}{\Gamma(\alpha)}|f(u, x(u))| d u d s\right. \\
& +\left|\rho_{1}(t)\right|\left[\int_{0}^{\xi} \int_{0}^{s}\left(e^{m_{2}(\xi-s)}-e^{m_{1}(\xi-s)}\right) \frac{(s-u)^{\alpha-1}}{\Gamma(\alpha)}|f(u, x(u))| d u d s\right. \\
& \left.+\sum_{i=1}^{n}\left|j_{i}\right| \int_{0}^{\eta_{i}} \int_{0}^{s}\left(e^{m_{2}\left(\eta_{i}-s\right)}-e^{m_{1}\left(\eta_{i}-s\right)}\right) \frac{(s-u)^{\alpha-1}}{\Gamma(\alpha)}|f(u, x(u))| d u d s\right] \\
& +\left|\rho_{2}(t)\right|\left[\int_{0}^{1} \int_{0}^{s}\left(e^{m_{2}(1-s)}-e^{m_{1}(1-s)}\right) \frac{(s-u)^{\alpha-1}}{\Gamma(\alpha)}|f(u, x(u))| d u d s\right. \\
& \left.\left.+\sum_{i=1}^{k}\left|\lambda_{i}\right| \int_{v_{i}}^{\sigma_{i}} \int_{0}^{s}\left(\frac{\left(e^{m_{2}\left(\sigma_{i}-s\right)}-1\right)}{m_{2}}-\frac{\left(e^{m_{1}\left(\sigma_{i}-s\right)}-1\right)}{m_{1}}\right) \frac{(s-u)^{\alpha-1}}{\Gamma(\alpha)}|f(u, x(u))| d u d s\right]\right\} \\
& \leq \frac{(\ell \bar{r}+M)}{|\hat{\delta}|} \sup _{t \in[0,1]}\left\{\int_{0}^{t}\left|e^{m_{2}(t-s)}-e^{m_{1}(t-s)}\right| \frac{s^{\alpha}}{\Gamma(\alpha+1)} d s\right. \\
& +\left|\rho_{1}(t)\right|\left[\int_{0}^{\xi}\left|e^{m_{2}(\xi-s)}-e^{m_{1}(\xi-s)}\right| \frac{s^{\alpha}}{\Gamma(\alpha+1)} d s\right. \\
& \left.+\sum_{i=1}^{n}\left|j_{i}\right| \int_{0}^{\eta_{i}}\left|e^{m_{2}\left(\eta_{i}-s\right)}-e^{m_{1}\left(\eta_{i}-s\right)}\right| \frac{s^{\alpha}}{\Gamma(\alpha+1)} d s\right] \\
& +\left|\rho_{2}(t)\right|\left[\int_{0}^{1}\left|e^{m_{2}(1-s)}-e^{m_{1}(1-s)}\right| \frac{s^{\alpha}}{\Gamma(\alpha+1)} d s\right. \\
& \left.\left.+\sum_{i=1}^{k}\left|\lambda_{i}\right| \int_{v_{i}}^{\sigma_{i}}\left|\frac{\left(e^{m_{2}\left(\sigma_{i}-s\right)}-1\right)}{m_{2}}-\frac{\left(e^{m_{1}\left(\sigma_{i}-s\right)}-1\right)}{m_{1}}\right| \frac{s^{\alpha}}{\Gamma(\alpha+1)} d s\right]\right\} \\
& \leq \frac{\left|\delta_{2}\right|(\ell \bar{r}+M)}{\left|\delta_{0} \hat{\delta}\right| \Gamma(\alpha+1)}\left\{\varepsilon+\widehat{\rho}_{1}\left[\xi^{\alpha}\left|m_{2}\left(1-e^{m_{1} \xi}\right)-m_{1}\left(1-e^{m_{2} \xi}\right)\right|\right.\right. \\
& \left.+\sum_{i=1}^{n}\left|j_{i}\right| \eta_{i}^{\alpha}\left|m_{2}\left(1-e^{m_{1} \eta_{i}}\right)-m_{1}\left(1-e^{m_{2} \eta_{i}}\right)\right|\right]+\widehat{\rho}_{2}\left[\left|m_{2}\left(1-e^{m_{1}}\right)-m_{1}\left(1-e^{m_{2}}\right)\right|\right.
\end{aligned}
$$




$$
\begin{aligned}
& +\frac{\left|\delta_{2}\right|}{\left|\delta_{0}\right|} \sum_{i=1}^{k}\left|\lambda_{i}\right| \sigma_{i}^{\alpha} \mid m_{2}^{2}\left(m_{1}\left(\sigma_{i}-v_{i}\right)-e^{m_{1}\left(\sigma_{i}-v_{i}\right)}+1\right) \\
& \left.\left.\quad-m_{1}^{2}\left(m_{2}\left(\sigma_{i}-v_{i}\right)-e^{m_{2}\left(\sigma_{i}-v_{i}\right)}+1\right) \mid\right]\right\} \\
& =(\ell \bar{r}+M) \phi \leq \bar{r},
\end{aligned}
$$

which clearly shows that $\mathcal{J} x \in B_{\bar{r}}$ for any $x \in B_{\bar{r}}$. Thus $\mathcal{J} B_{\bar{r}} \subset B_{\bar{r}}$. Now, for $x, y \in \mathcal{C}$ and for each $t \in[0,1]$, we have

$$
\begin{aligned}
& \|(\mathcal{J} x)-(\mathcal{J} y)\| \\
& \leq \frac{1}{|\hat{\delta}|} \sup _{t \in[0,1]}\left\{\int_{0}^{t} \int_{0}^{s}\left(e^{m_{2}(t-s)}-e^{m_{1}(t-s)}\right) \frac{(s-u)^{\alpha-1}}{\Gamma(\alpha)}|f(u, x(u))-f(u, y(u))| d u d s\right. \\
& +\left|\rho_{1}(t)\right|\left[\int_{0}^{\xi} \int_{0}^{s}\left(e^{m_{2}(\xi-s)}-e^{m_{1}(\xi-s)}\right) \frac{(s-u)^{\alpha-1}}{\Gamma(\alpha)}|f(u, x(u))-f(u, y(u))| d u d s\right. \\
& \left.+\sum_{i=1}^{n}\left|j_{i}\right| \int_{0}^{\eta_{i}} \int_{0}^{s}\left(e^{m_{2}\left(\eta_{i}-s\right)}-e^{m_{1}\left(\eta_{i}-s\right)}\right) \frac{(s-u)^{\alpha-1}}{\Gamma(\alpha)}|f(u, x(u))-f(u, y(u))| d u d s\right] \\
& +\left|\rho_{2}(t)\right|\left[\int_{0}^{1} \int_{0}^{s}\left(e^{m_{2}(1-s)}-e^{m_{1}(1-s)}\right) \frac{(s-u)^{\alpha-1}}{\Gamma(\alpha)}|f(u, x(u))-f(u, y(u))| d u d s\right. \\
& +\sum_{i=1}^{k}\left|\lambda_{i}\right| \int_{v_{i}}^{\sigma_{i}} \int_{0}^{s}\left(\frac{\left(e^{m_{2}\left(\sigma_{i}-s\right)}-1\right)}{m_{2}}-\frac{\left(e^{m_{1}\left(\sigma_{i}-s\right)}-1\right)}{m_{1}}\right) \\
& \left.\left.\times \frac{(s-u)^{\alpha-1}}{\Gamma(\alpha)}|f(u, x(u))-f(u, y(u))| d u d s\right]\right\} \\
& \leq \frac{\ell}{|\hat{\delta}|} \sup _{t \in[0,1]}\left\{\int_{0}^{t}\left|e^{m_{2}(t-s)}-e^{m_{1}(t-s)}\right| \frac{s^{\alpha}}{\Gamma(\alpha+1)} d s\right. \\
& +\left|\rho_{1}(t)\right|\left[\int_{0}^{\xi}\left|e^{m_{2}(\xi-s)}-e^{m_{1}(\xi-s)}\right| \frac{s^{\alpha}}{\Gamma(\alpha+1)} d s\right. \\
& \left.+\sum_{i=1}^{n}\left|j_{i}\right| \int_{0}^{\eta_{i}}\left|e^{m_{2}\left(\eta_{i}-s\right)}-e^{m_{1}\left(\eta_{i}-s\right)}\right| \frac{s^{\alpha}}{\Gamma(\alpha+1)} d s\right] \\
& +\left|\rho_{2}(t)\right|\left[\int_{0}^{1}\left|e^{m_{2}(1-s)}-e^{m_{1}(1-s)}\right| \frac{s^{\alpha}}{\Gamma(\alpha+1)} d s\right. \\
& \left.\left.+\sum_{i=1}^{k}\left|\lambda_{i}\right| \int_{v_{i}}^{\sigma_{i}}\left|\frac{\left(e^{m_{2}\left(\sigma_{i}-s\right)}-1\right)}{m_{2}}-\frac{\left(e^{m_{1}\left(\sigma_{i}-s\right)}-1\right)}{m_{1}}\right| \frac{s^{\alpha}}{\Gamma(\alpha+1)} d s\right]\right\}\|x-y\| \\
& \leq \frac{\left|\delta_{2}\right| \ell}{\left|\delta_{0} \hat{\delta}\right| \Gamma(\alpha+1)}\left\{\varepsilon+\widehat{\rho}_{1}\left[\xi^{\alpha}\left|m_{2}\left(1-e^{m_{1} \xi}\right)-m_{1}\left(1-e^{m_{2} \xi}\right)\right|\right.\right. \\
& \left.+\sum_{i=1}^{n}\left|j_{i}\right| \eta_{i}^{\alpha}\left|m_{2}\left(1-e^{m_{1} \eta_{i}}\right)-m_{1}\left(1-e^{m_{2} \eta_{i}}\right)\right|\right]+\widehat{\rho}_{2}\left[\left|m_{2}\left(1-e^{m_{1}}\right)-m_{1}\left(1-e^{m_{2}}\right)\right|\right. \\
& +\frac{\left|\delta_{2}\right|}{\left|\delta_{0}\right|} \sum_{i=1}^{k}\left|\lambda_{i}\right| \sigma_{i}^{\alpha} \mid m_{2}^{2}\left(m_{1}\left(\sigma_{i}-v_{i}\right)-e^{m_{1}\left(\sigma_{i}-v_{i}\right)}+1\right)
\end{aligned}
$$




$$
\begin{aligned}
& \left.\left.\quad-m_{1}^{2}\left(m_{2}\left(\sigma_{i}-v_{i}\right)-e^{m_{2}\left(\sigma_{i}-v_{i}\right)}+1\right) \mid\right]\right\}\|x-y\| \\
& =\ell \phi\|x-y\|,
\end{aligned}
$$

where $\phi$ is given by (3.2) and depends only on the parameters involved in the problem. In view of the condition $\ell<1 / \phi$, it follows that $\mathcal{J}$ is a contraction. Thus, by the contraction mapping principle (Banach fixed point theorem), the problem (1.1)-(1.2) with $\delta_{1}{ }^{2}-4 \delta_{0} \delta_{2}>$ 0 has a unique solution on $[0,1]$. This completes the proof.

The next existence result is based on Leray-Schauder nonlinear alternative.

Theorem 3.5 (Nonlinear alternative for single valued maps [27]) Let C be a closed, convex subset of be a Banach space $E$ and $U$ an open subset of $C$ with $0 \in U$. Suppose that $F: \bar{U} \rightarrow$ $C$ is a continuous, compact (that is, $F(\bar{U})$ is a relatively compact subset of $C$ ) map. Then either

(i) F has a fixed point in $\bar{U}$, or

(ii) there is a $u \in \partial U$ (the boundary of $U$ in $C$ ) and $\epsilon \in(0,1)$ with $u=\epsilon F(u$ ).

We need the following assumptions:

$\left(\mathrm{H}_{1}\right)$ There exist a function $g \in C\left([0,1], \mathbb{R}^{+}\right)$, and a nondecreasing function $Q: \mathbb{R}^{+} \rightarrow \mathbb{R}^{+}$ such that $|f(t, y)| \leq g(t) Q(\|y\|), \forall(t, y) \in[0,1] \times \mathbb{R}$.

$\left(\mathrm{H}_{2}\right)$ There exists a constant $K>0$ such that

$$
\frac{K}{\|g\| Q(K) \phi}>1
$$

Theorem 3.6 Let $f:[0,1] \times \mathbb{R} \rightarrow \mathbb{R}$ be a continuous function and suppose assumptions $\left(\mathrm{H}_{1}\right)$ and $\left(\mathrm{H}_{2}\right)$ are satisfied. Then the problem (1.1)-(1.2), with $\delta_{1}{ }^{2}-4 \delta_{0} \delta_{2}>0$, has at least one solution on $[0,1]$.

Proof Consider the operator $\mathcal{J}: \mathcal{C} \rightarrow \mathcal{C}$ defined by (3.1). We show that $\mathcal{J}$ maps bounded sets into bounded sets in $\mathcal{C}=C([0,1], \mathbb{R})$. For a positive number $\zeta$, let $\mathcal{B}_{\zeta}=\{x \in \mathcal{C}:\|x\| \leq \zeta\}$ be a bounded set in $\mathcal{C}$. Then we have

$$
\begin{aligned}
\|\mathcal{J}(x)\|= & \sup _{t \in[0,1]}|\mathcal{J}(x)(t)| \\
\leq & \frac{1}{|\hat{\delta}|} \sup _{t \in[0,1]}\left\{\int_{0}^{t} \int_{0}^{s}\left(e^{m_{2}(t-s)}-e^{m_{1}(t-s)}\right) \frac{(s-u)^{\alpha-1}}{\Gamma(\alpha)}|f(u, x(u))| d u d s\right. \\
& +\left|\rho_{1}(t)\right|\left[\int_{0}^{\xi} \int_{0}^{s}\left(e^{m_{2}(\xi-s)}-e^{m_{1}(\xi-s)}\right) \frac{(s-u)^{\alpha-1}}{\Gamma(\alpha)}|f(u, x(u))| d u d s\right. \\
& \left.+\sum_{i=1}^{n}\left|j_{i}\right| \int_{0}^{\eta_{i}} \int_{0}^{s}\left(e^{m_{2}\left(\eta_{i}-s\right)}-e^{m_{1}\left(\eta_{i}-s\right)}\right) \frac{(s-u)^{\alpha-1}}{\Gamma(\alpha)}|f(u, x(u))| d u d s\right] \\
& +\left|\rho_{2}(t)\right|\left[\int_{0}^{1} \int_{0}^{s}\left(e^{m_{2}(1-s)}-e^{m_{1}(1-s)}\right) \frac{(s-u)^{\alpha-1}}{\Gamma(\alpha)}|f(u, x(u))| d u d s\right.
\end{aligned}
$$




$$
\begin{aligned}
& +\sum_{i=1}^{k}\left|\lambda_{i}\right| \int_{v_{i}}^{\sigma_{i}} \int_{0}^{s}\left(\frac{\left(e^{m_{2}\left(\sigma_{i}-s\right)}-1\right)}{m_{2}}-\frac{\left(e^{m_{1}\left(\sigma_{i}-s\right)}-1\right)}{m_{1}}\right) \\
& \left.\left.\times \frac{(s-u)^{\alpha-1}}{\Gamma(\alpha)}|f(u, x(u))| d u d s\right]\right\} \\
\leq & \frac{\|g\| Q(\zeta)}{|\hat{\delta}|} \sup _{t \in[0,1]}\left\{\int_{0}^{t}\left|e^{m_{2}(t-s)}-e^{m_{1}(t-s)}\right| \frac{s^{\alpha}}{\Gamma(\alpha+1)} d s\right. \\
& +\left|\rho_{1}(t)\right|\left[\int_{0}^{\xi}\left|e^{m_{2}(\xi-s)}-e^{m_{1}(\xi-s)}\right| \frac{s^{\alpha}}{\Gamma(\alpha+1)} d s\right. \\
& \left.+\sum_{i=1}^{n} j_{i} \int_{0}^{\eta_{i}}\left|e^{m_{2}\left(\eta_{i}-s\right)}-e^{m_{1}\left(\eta_{i}-s\right)}\right| \frac{s^{\alpha}}{\Gamma(\alpha+1)} d s\right] \\
& +\left|\rho_{2}(t)\right|\left[\int_{0}^{1}\left|e^{m_{2}(1-s)}-e^{m_{1}(1-s)}\right| \frac{s^{\alpha}}{\Gamma(\alpha+1)} d s\right. \\
& \left.\left.+\sum_{i=1}^{k}\left|\lambda_{i}\right| \int_{v_{i}}^{\sigma_{i}}\left|\frac{\left(e^{m_{2}\left(\sigma_{i}-s\right)}-1\right)}{m_{2}}-\frac{\left(e^{m_{1}\left(\sigma_{i}-s\right)}-1\right)}{m_{1}}\right| \frac{s^{\alpha}}{\Gamma(\alpha+1)} d s\right]\right\} \\
\leq & \frac{\left|\delta_{2}\right|\|g\| \mid Q(\zeta)}{\left|\delta_{0} \hat{\delta}\right| \Gamma(\alpha+1)}\left\{\varepsilon+\widehat{\rho}_{1}\left[\xi^{\alpha}\left|m_{2}\left(1-e^{m_{1} \xi}\right)-m_{1}\left(1-e^{m_{2} \xi}\right)\right|\right.\right. \\
& \left.+\sum_{i=1}^{n}\left|j_{i}\right| \eta_{i}^{\alpha}\left|m_{2}\left(1-e^{m_{1} \eta_{i}}\right)-m_{1}\left(1-e^{m_{2} \eta_{i}}\right)\right|\right] \\
& +\widehat{\rho}_{2}\left[\left|m_{2}\left(1-e^{m_{1}}\right)-m_{1}\left(1-e^{m_{2}}\right)\right|\right. \\
\left|\delta_{0}\right| & \left.\sum_{i=1}^{2}\left(m_{2}\left(\sigma_{i}-v_{i}\right)-e^{m_{2}\left(\sigma_{i}-v_{i}\right)}+1\right) \mid\right],
\end{aligned}
$$

which yields

$$
\begin{aligned}
\|\mathcal{J} x\| \leq & \frac{\left|\delta_{2}\right|\|g\| Q(\zeta)}{\left|\delta_{0} \hat{\delta}\right| \Gamma(\alpha+1)}\left\{\varepsilon+\widehat{\rho}_{1} \xi^{\alpha}\left|m_{2}\left(1-e^{m_{1} \xi}\right)-m_{1}\left(1-e^{m_{2} \xi}\right)\right|\right. \\
& +\sum_{i=1}^{n}\left|j_{i}\right| \eta_{i}^{\alpha}\left|m_{2}\left(1-e^{m_{1} \eta_{i}}\right)-m_{1}\left(1-e^{m_{2} \eta_{i}}\right)\right| \\
& +\widehat{\rho}_{2}\left[\left|m_{2}\left(1-e^{m_{1}}\right)-m_{1}\left(1-e^{m_{2}}\right)\right|\right. \\
& +\frac{\left|\delta_{2}\right|}{\left|\delta_{0}\right|} \sum_{i=1}^{k}\left|\lambda_{i}\right| \sigma_{i}^{\alpha} \mid m_{2}^{2}\left(m_{1}\left(\sigma_{i}-v_{i}\right)-e^{m_{1}\left(\sigma_{i}-v_{i}\right)}+1\right) \\
& \left.\left.-m_{1}^{2}\left(m_{2}\left(\sigma_{i}-v_{i}\right)-e^{m_{2}\left(\sigma_{i}-v_{i}\right)}+1\right) \mid\right]\right\} .
\end{aligned}
$$


Next we show that $\mathcal{J}$ maps bounded sets into equicontinuous sets of $\mathcal{C}$. Let $t_{1}, t_{2} \in[0,1]$ with $t_{1}<t_{2}$ and $y \in \mathcal{B}_{\zeta}$, where $\mathcal{B}_{\zeta}$ is a bounded set of $\mathcal{C}$. Then we obtain

$$
\begin{aligned}
& \left|(\mathcal{J} x)\left(t_{2}\right)-(\mathcal{J} x)\left(t_{1}\right)\right| \\
& \leq \frac{1}{|\hat{\delta}|}\left\{\mid \int_{0}^{t_{1}} \int_{0}^{s}\left[\left(e^{m_{2}\left(t_{2}-s\right)}-e^{m_{1}\left(t_{2}-s\right)}\right)-\left(e^{m_{2}\left(t_{1}-s\right)}-e^{m_{1}\left(t_{1}-s\right)}\right)\right]\right. \\
& \times \frac{(s-u)^{\alpha-1}}{\Gamma(\alpha)} f(u, x(u)) d u d s \\
& +\int_{t_{1}}^{t_{2}} \int_{0}^{s}\left(e^{m_{2}\left(t_{2}-s\right)}-e^{m_{1}\left(t_{2}-s\right)}\right) \frac{(s-u)^{\alpha-1}}{\Gamma(\alpha)} f(u, x(u)) d u d s \mid \\
& +\left|\rho_{1}\left(t_{2}\right)-\rho_{1}\left(t_{1}\right)\right| \int_{0}^{\xi} \int_{0}^{s}\left(e^{m_{2}(\xi-s)}-e^{m_{1}(\xi-s)}\right) \frac{(s-u)^{\alpha-1}}{\Gamma(\alpha)}|f(u, x(u))| d u d s \\
& +\sum_{i=1}^{n}\left|j_{i}\right| \int_{0}^{\eta_{i}} \int_{0}^{s}\left[\left(e^{m_{2}\left(\eta_{i}-s\right)}-e^{m_{1}\left(\eta_{i}-s\right)}\right) \frac{(s-u)^{\alpha-1}}{\Gamma(\alpha)}|f(u, x(u))| d u d s\right] \\
& +\left|\rho_{2}\left(t_{2}\right)-\rho_{2}\left(t_{1}\right)\right|\left[\int_{0}^{1} \int_{0}^{s}\left(e^{m_{2}(1-s)}-e^{m_{1}(1-s)}\right) \frac{(s-u)^{\alpha-1}}{\Gamma(\alpha)}|f(u, x(u))| d u d s\right. \\
& +\sum_{i=1}^{k}\left|\lambda_{i}\right| \int_{v_{i}}^{\sigma_{i}} \int_{0}^{s}\left(\frac{\left(e^{m_{2}\left(\sigma_{i}-s\right)}-1\right)}{m_{2}}-\frac{\left(e^{m_{1}\left(\sigma_{i}-s\right)}-1\right)}{m_{1}}\right) \\
& \left.\left.\times \frac{(s-u)^{\alpha-1}}{\Gamma(\alpha)}|f(u, x(u))| d u d s\right]\right\} \\
& \leq \frac{\left|\delta_{2}\right|\|g\| Q(\zeta)}{\left|\delta_{0} \hat{\delta}\right| \Gamma(\alpha+1)}\left\{\left(t_{1}^{\alpha}-t_{2}^{\alpha}\right) \mid m_{1}\left(1-e^{m_{2}\left(t_{2}-t_{1}\right)}\right)-m_{2}\left(1-e^{m_{1}\left(t_{2}-t_{1}\right)} \mid\right)\right. \\
& +t_{1}^{\alpha} \mid m_{1}\left(e^{m_{2} t_{2}}-e^{m_{2} t_{1}}\right)-m_{2}\left(e^{m_{1} t_{2}}-e^{m_{1} t_{1}} \mid\right) \\
& +\left|\rho_{1}\left(t_{2}\right)-\rho_{1}\left(t_{1}\right)\right|\left[\xi^{\alpha}\left|m_{2}\left(1-e^{m_{1} \xi}\right)-m_{1}\left(1-e^{m_{2} \xi}\right)\right|\right. \\
& \left.+\sum_{i=1}^{n}\left|j_{i}\right| \eta_{i}^{\alpha}\left|m_{2}\left(1-e^{m_{1} \eta_{i}}\right)-m_{1}\left(1-e^{m_{2} \eta_{i}}\right)\right|\right] \\
& +\left|\rho_{2}\left(t_{2}\right)-\rho_{2}\left(t_{1}\right)\right|\left[\left|m_{2}\left(1-e^{m_{1}}\right)-m_{1}\left(1-e^{m_{2}}\right)\right|\right. \\
& +\frac{\left|\delta_{2}\right|}{\left|\delta_{0}\right|} \sum_{i=1}^{k}\left|\lambda_{i}\right| \sigma_{i}^{\alpha} \mid m_{1}^{2}\left(m_{2}\left(\sigma_{i}-v_{i}\right)-e^{m_{2}\left(\sigma_{i}-v_{i}\right)}+1\right) \\
& \left.\left.-m_{2}^{2}\left(m_{1}\left(\sigma_{i}-v_{i}\right)-e^{m_{1}\left(\sigma_{i}-v_{i}\right)}+1\right) \mid\right]\right\}
\end{aligned}
$$

which tends to zero independently of $x \in \mathcal{B}_{\zeta}$ as $t_{2}-t_{1} \rightarrow 0$. As $\mathcal{J}$ satisfies the above assumptions, it follows by the Arzelá-Ascoli theorem that $\mathcal{J}: \mathcal{C} \rightarrow \mathcal{C}$ is completely continuous.

The result will follow from the Leray-Schauder nonlinear alternative once it is shown that the set of all solutions to the equation $x=\theta \mathcal{J} x$ is bounded for $\theta \in[0,1]$. For that, let 
$x$ be a solution of $x=\theta \mathcal{J} x$ for $\theta \in[0,1]$. Then, for $t \in[0,1]$, we have

$$
\begin{aligned}
& |x(t)|=|\theta \mathcal{J} x(t)| \\
& \leq \frac{1}{|\hat{\delta}|} \sup _{t \in[0,1]}\left\{\int_{0}^{t} \int_{0}^{s}\left(e^{m_{2}(t-s)}-e^{m_{1}(t-s)}\right) \frac{(s-u)^{\alpha-1}}{\Gamma(\alpha)}|f(u, x(u))| d u d s\right. \\
& +\left|\rho_{1}(t)\right|\left[\int_{0}^{\xi} \int_{0}^{s}\left(e^{m_{2}(\xi-s)}-e^{m_{1}(\xi-s)}\right) \frac{(s-u)^{\alpha-1}}{\Gamma(\alpha)}|f(u, x(u))| d u d s\right. \\
& \left.+\sum_{i=1}^{n}\left|j_{i}\right| \int_{0}^{\eta_{i}} \int_{0}^{s}\left(e^{m_{2}\left(\eta_{i}-s\right)}-e^{m_{1}\left(\eta_{i}-s\right)}\right) \frac{(s-u)^{\alpha-1}}{\Gamma(\alpha)}|f(u, x(u))| d u d s\right] \\
& +\left|\rho_{2}(t)\right|\left[\int_{0}^{1} \int_{0}^{s}\left(e^{m_{2}(1-s)}-e^{m_{1}(1-s)}\right) \frac{(s-u)^{\alpha-1}}{\Gamma(\alpha)}|f(u, x(u))| d u d s\right. \\
& +\sum_{i=1}^{k}\left|\lambda_{i}\right| \int_{v_{i}}^{\sigma_{i}} \int_{0}^{s}\left(\frac{\left(e^{m_{2}\left(\sigma_{i}-s\right)}-1\right)}{m_{2}}-\frac{\left(e^{m_{1}\left(\sigma_{i}-s\right)}-1\right)}{m_{1}}\right) \\
& \left.\left.\times \frac{(s-u)^{\alpha-1}}{\Gamma(\alpha)}|f(u, x(u))| d u d s\right]\right\} \\
& \leq \frac{\|g\| Q(\|x\|)}{|\hat{\delta}|} \sup _{t \in[0,1]}\left\{\int_{0}^{t}\left|e^{m_{2}(t-s)}-e^{m_{1}(t-s)}\right| \frac{s^{\alpha}}{\Gamma(\alpha+1)} d s\right. \\
& +\left|\rho_{1}(t)\right|\left[\int_{0}^{\xi}\left|e^{m_{2}(\xi-s)}-e^{m_{1}(\xi-s)}\right| \frac{s^{\alpha}}{\Gamma(\alpha+1)} d s\right. \\
& \left.+\sum_{i=1}^{n}\left|j_{i}\right| \int_{0}^{\eta_{i}}\left|e^{m_{2}\left(\eta_{i}-s\right)}-e^{m_{1}\left(\eta_{i}-s\right)}\right| \frac{s^{\alpha}}{\Gamma(\alpha+1)} d s\right] \\
& +\left|\rho_{2}(t)\right|\left[\int_{0}^{1}\left|e^{m_{2}(1-s)}-e^{m_{1}(1-s)}\right| \frac{s^{\alpha}}{\Gamma(\alpha+1)} d s\right. \\
& \left.\left.+\sum_{i=1}^{k}\left|\lambda_{i}\right| \int_{v_{i}}^{\sigma_{i}}\left|\frac{\left(e^{m_{2}\left(\sigma_{i}-s\right)}-1\right)}{m_{2}}-\frac{\left(e^{m_{1}\left(\sigma_{i}-s\right)}-1\right)}{m_{1}}\right| \frac{s^{\alpha}}{\Gamma(\alpha+1)} d s\right]\right\} \\
& \leq \frac{\left|\delta_{2}\right|\|g\| Q(\|x\|)}{\left|\delta_{0} \hat{\delta}\right| \Gamma(\alpha+1)}\left\{\varepsilon+\widehat{\rho}_{1}\left[\xi^{\alpha}\left|m_{2}\left(1-e^{m_{1} \xi}\right)-m_{1}\left(1-e^{m_{2} \xi}\right)\right|\right.\right. \\
& \left.+\sum_{i=1}^{n}\left|j_{i}\right| \eta_{i}^{\alpha}\left|m_{2}\left(1-e^{m_{1} \eta_{i}}\right)-m_{1}\left(1-e^{m_{2} \eta_{i}}\right)\right|\right] \\
& +\widehat{\rho}_{2}\left[\left|m_{2}\left(1-e^{m_{1}}\right)-m_{1}\left(1-e^{m_{2}}\right)\right|\right. \\
& +\frac{\left|\delta_{2}\right|}{\left|\delta_{0}\right|} \sum_{i=1}^{k}\left|\lambda_{i}\right| \sigma_{i}^{\alpha} \mid m_{1}^{2}\left(m_{2}\left(\sigma_{i}-v_{i}\right)-e^{m_{2}\left(\sigma_{i}-v_{i}\right)}+1\right) \\
& \left.\left.-m_{2}^{2}\left(m_{1}\left(\sigma_{i}-v_{i}\right)-e^{m_{1}\left(\sigma_{i}-v_{i}\right)}+1\right) \mid\right]\right\} \\
& =\|g\| Q(\|x\|) \phi \text {, }
\end{aligned}
$$


which implies that

$$
\frac{\|x\|}{\|g\| Q(\|x\|) \phi} \leq 1
$$

In view of $\left(\mathrm{H}_{2}\right)$, there is no solution $x$ such that $\|x\| \neq K$. Let us set

$$
U=\{x \in \mathcal{C}:\|x\|<K\} .
$$

The operator $\mathcal{J}: \bar{U} \rightarrow \mathcal{C}$ is continuous and completely continuous. From the choice of $U$, there is no $u \in \partial U$ such that $u=\theta \mathcal{J}(u)$ for some $\theta \in(0,1)$. Consequently, by the nonlinear alternative of Leray-Schauder type [27], we deduce that $\mathcal{J}$ has a fixed point $u \in \bar{U}$ which is a solution of the problem (1.1)-(1.2) with $\delta_{1}{ }^{2}-4 \delta_{0} \delta_{2}>0$. The proof is completed.

Example 3.7 Consider the following multi-term fractional differential equation

$$
\left(2^{\mathrm{c}} D^{7 / 3}+3^{\mathrm{c}} D^{4 / 3}+{ }^{\mathrm{c}} D^{1 / 3}\right) x(t)=\frac{A}{4(1+t)^{2}} \tan ^{-1} x+\sin (t+3), \quad 0<t<1,
$$

subject to the boundary conditions

$$
x(0)=0, \quad x(1 / 6)=2 x(1 / 5)+x(2 / 5), \quad x(1)=\int_{1 / 4}^{3 / 5} x(s) d s+3 \int_{2 / 3}^{4 / 5} x(s) d s .
$$

Here $\alpha=1 / 3, \xi=1 / 6, \eta_{1}=1 / 5, \eta_{2}=2 / 5, v_{1}=1 / 4, v_{2}=2 / 3, \sigma_{1}=3 / 5, \sigma_{2}=4 / 5 j_{1}=2$, $j_{2}=1, \lambda_{1}=1, \lambda_{2}=3$ and

$$
f(t, x)=\frac{A}{4(1+t)^{2}} \tan ^{-1} x+\sin (t+3)
$$

$A$ is positive number. Clearly, $\delta_{1}^{2}-4 \delta_{0} \delta_{2}=1>0,|f(t, x)-f(t, y)| \leq \ell|x-y|$ with $\ell=A / 4$. Using the given values, we find that $\phi \approx 0.66348$ and $\phi_{1} \approx 0.49011$. Further, we have that $|f(t, x)| \leq \frac{\pi A}{8(1+t)^{2}}+\sin (t+3)=\vartheta(t)$ and $\ell \phi_{1}<1$ when $A<8.16143$. As all the conditions of Theorem 3.2 are satisfied, the conclusion of Theorem 3.2 applies to the problem (3.7)(3.8). On the other hand, as $\ell \phi<1$ for $A<6.02882$, there exists a unique solution for the problem (3.7)-(3.8) on $[0,1]$ by Theorem 3.4 .

Example 3.8 Consider the multi-term fractional differential equation:

$$
\left(2^{\mathrm{c}} D^{7 / 3}+3^{\mathrm{c}} D^{4 / 3}+{ }^{\mathrm{c}} D^{1 / 3}\right) x(t)=\frac{2}{\sqrt{t^{2}+64}}\left(|x|\left(\frac{|x|}{|x|+1}\right)+\frac{1}{5}\right), \quad 0<t<1,
$$

supplemented with the boundary conditions (3.8).

Observe that $\delta_{1}^{2}-4 \delta_{2} \delta_{0}=1>0$ and $|f(t, x)| \leq g(t) Q(\|x\|)$ with $g(t)=\frac{2}{\sqrt{t^{2}+64}}$ and $Q(\|x\|)=$ $\|x\|+\frac{1}{5}$. Due to condition $\left(\mathrm{H}_{2}\right)$, using $\phi \approx 0.66348$, we find that $K>0.15908$. Thus, by the conclusion of Theorem 3.6, there exists at least one solution for the equation (3.9) with the boundary conditions (3.8). 
4 Existence results for problem (1.1)-(1.2) with $\delta_{1}^{2}-\mathbf{4} \delta_{0} \delta_{2}=0$

In view of Lemma 2.7, we can transform problem (1.1)-(1.2) with $\delta_{1}^{2}-4 \delta_{0} \delta_{2}=0$ into an equivalent fixed point problem as

$$
x=\mathcal{H} x,
$$

where the operator $\mathcal{H}: \mathcal{C} \rightarrow \mathcal{C}$ is defined by

$$
\begin{aligned}
(\mathcal{H} x)(t)= & \frac{1}{\delta_{2}}\left\{\int_{0}^{t} \int_{0}^{s}(t-s) e^{m(t-s)} \frac{(s-u)^{\alpha-1}}{\Gamma(\alpha)} f(u, x(u)) d u d s\right. \\
& +\chi_{1}(t)\left[\int_{0}^{\xi} \int_{0}^{s}(\xi-s) e^{m(\xi-s)} \frac{(s-u)^{\alpha-1}}{\Gamma(\alpha)} f(u, x(u)) d u d s\right. \\
& \left.-\sum_{i=1}^{n} j_{i} \int_{0}^{\eta_{i}} \int_{0}^{s}\left(\eta_{i}-s\right) e^{m\left(\eta_{i}-s\right)} \frac{(s-u)^{\alpha-1}}{\Gamma(\alpha)} f(u, x(u)) d u d s\right] \\
& +\chi_{2}(t)\left[\int_{0}^{1} \int_{0}^{s}(1-s) e^{m(1-s)} \frac{(s-u)^{q-1}}{\Gamma(q)} f(u, x(u)) d u d s\right. \\
& -\sum_{i=1}^{k} \lambda_{i} \int_{v_{i}}^{\sigma_{i}} \int_{0}^{s}\left(\frac{m(\sigma-s) e^{m(\sigma-s)}-e^{m(\sigma-s)}+1}{m^{2}}\right) \\
& \left.\left.\times \frac{(s-u)^{q-1}}{\Gamma(q)} f(u, x(u)) d u d s\right]\right\},
\end{aligned}
$$

$\chi_{1}(t)$ and $\chi_{2}(t)$ are defined by (2.12). Moreover, we set

$$
\begin{aligned}
\widehat{\chi}_{1}= & \max _{t \in[0,1]}\left|\chi_{1}(t)\right|, \quad \widehat{\chi}_{2}=\max _{t \in[0,1]}\left|\chi_{2}(t)\right|, \\
\beta= & \frac{1}{\left|\delta_{2}\right| m^{2} \Gamma(\alpha+1)}\left\{\left(1+\widehat{\chi}_{2}\right)\left|(m-1) e^{m}+1\right|\right. \\
& +\widehat{\chi}_{1}\left[\xi^{\alpha}\left|(m \xi-1) e^{m \xi}+1\right|+\sum_{i=1}^{n}\left|j_{i}\right| \eta_{i}^{\alpha}\left|\left(m \eta_{i}-1\right) e^{m \eta_{i}}+1\right|\right] \\
& \left.+\widehat{\chi}_{2} \frac{\sum_{i=1}^{k}\left|\lambda_{i}\right| \sigma_{i}^{\alpha}}{|m|}\left|m\left(\sigma_{i}-v_{i}\right)\left(e^{m\left(\sigma_{i}-v_{i}\right)}+1\right)+2\left(1-e^{m\left(\sigma_{i}-v_{i}\right)}\right)\right|\right\}, \\
\beta_{1}= & \beta-\frac{\left|(m-1) e^{m}+1\right|}{\left|\delta_{2}\right| m^{2} \Gamma(\alpha+1)} .
\end{aligned}
$$

Now we present existence results for the problem (1.1)-(1.2) with $\delta_{1}^{2}-4 \delta_{0} \delta_{2}=0$ without proof. One can complete the proofs for these results following the arguments used in the previous section.

Theorem 4.1 Let $f:[0,1] \times \mathbb{R} \rightarrow \mathbb{R}$ be a continuous function satisfying conditions $\left(\mathrm{A}_{1}\right)$ and $\left(\mathrm{A}_{2}\right)$. Then the problem (1.1)-(1.2), with $\delta_{1}^{2}-4 \delta_{0} \delta_{2}=0$, has at least one solution on $[0,1]$ if $\ell \beta_{1}<1$, where $\beta_{1}$ is given by $(4.2)$. 
Theorem 4.2 Assume that $f:[0,1] \times \mathbb{R} \rightarrow \mathbb{R}$ is a continuous function and condition $\left(\mathrm{A}_{1}\right)$ is satisfied. Then there exists a unique solution for problem (1.1)-(1.2), with $\delta_{1}^{2}-4 \delta_{0} \delta_{2}=0$, on $[0,1]$ if $\ell<1 / \beta$, where $\beta$ is given by $(4.2)$.

Theorem 4.3 Let $f:[0,1] \times \mathbb{R} \rightarrow \mathbb{R}$ be a continuous function. In addition, suppose that $\left(\mathrm{H}_{1}\right)$ and the following condition hold:

$\left(\mathrm{H}_{2}^{\prime}\right)$ There exists a constant $K_{1}>0$ such that $\frac{K_{1}}{\|g\| Q\left(K_{1}\right) \beta}>1$, where $\beta$ is defined by (4.2). Then the problem (1.1)-(1.2), with $\delta_{1}^{2}-4 \delta_{0} \delta_{2}=0$, has at least one solution on $[0,1]$.

Example 4.4 Let us consider the multi-term fractional differential equation

$$
\left({ }^{\mathrm{c}} D^{7 / 3}+2^{\mathrm{c}} D^{4 / 3}+{ }^{\mathrm{c}} D^{1 / 3}\right) x(t)=\left(\frac{|x|}{1+|x|}+t\right) \frac{B}{2 \sqrt{t^{2}+4}}+\cos t, \quad 0<t<1,
$$

supplemented with the boundary conditions (3.8), where

$$
f(t, x)=\left(\frac{|x|}{1+|x|}+t\right) \frac{B}{2 \sqrt{t^{2}+4}}+\cos t
$$

and $B$ is positive number.

Obviously, $\delta_{1}^{2}-4 \delta_{0} \delta_{2}=0$, and $|f(t, x)-f(t, y)| \leq \ell|x-y|$ with $\ell=B / 4$. Using the given values, we find that $\beta \approx 0.39636$ and $\beta_{1} \approx 0.10045$. It is easy to check that $|f(t, x)| \leq$ $\frac{B(1+t)}{2 \sqrt{t^{2}+4}}+\cos t=\vartheta(t)$ and $\ell \beta_{1}<1$ when $B<39.82081$. As all the condition of Theorem 4.1 are satisfied, equation (4.3) with the boundary data (3.8) has at least one solution on $[0,1]$. On the other hand, $\ell \beta<1$ whenever $B<10.091836$, so there exists a unique solution for equation (4.3) with the boundary data $(3.8)$ on $[0,1]$ by Theorem 4.2 .

\section{Existence results for problem (1.1)-(1.2) with $\delta_{1}^{2}-\mathbf{4} \delta_{0} \delta_{2}<0$}

By Lemma 2.8, the fixed point problem equivalent to the problem (1.1)-(1.2) with $\delta_{1}^{2}-$ $4 \delta_{0} \delta_{2}<0$ can be written as

$$
x=\mathcal{K} x
$$

where the operator $\mathcal{K}: \mathcal{C} \rightarrow \mathcal{C}$ is defined by

$$
\begin{aligned}
(\mathcal{K} x)(t)= & \frac{1}{\delta_{2} b}\left\{\int_{0}^{t} \int_{0}^{s} e^{-a(t-s)} \sin b(t-s) \frac{(s-u)^{\alpha-1}}{\Gamma(\alpha)} f(u, x(u)) d u d s\right. \\
& +\tau_{1}(t)\left[\int_{0}^{\xi} \int_{0}^{s} e^{-a(\xi-s)} \sin b(\xi-s) \frac{(s-u)^{\alpha-1}}{\Gamma(\alpha)} f(u, x(u)) d u d s\right. \\
& \left.-\sum_{i=1}^{n} j_{i} \int_{0}^{\eta_{i}} \int_{0}^{s} e^{-a\left(\eta_{i}-s\right)} \sin b\left(\eta_{i}-s\right) \frac{(s-u)^{\alpha-1}}{\Gamma(\alpha)} f(u, x(u)) d u d s\right] \\
& +\tau_{2}(t)\left[\int_{0}^{1} \int_{0}^{s} e^{-a(1-s)} \sin b(1-s) \frac{(s-u)^{q-1}}{\Gamma(q)} f(u, x(u)) d u d s\right.
\end{aligned}
$$




$$
\begin{aligned}
& -\frac{\sum_{i=1}^{k} \lambda_{i}}{a^{2}+b^{2}} \int_{v_{i}}^{\sigma_{i}} \int_{0}^{s}\left(b-b e^{-a(\sigma-s)} \cos b(\sigma-s)\right. \\
& \left.\left.\left.-a e^{-a(\sigma-s)} \sin b(\sigma-s)\right) \frac{(s-u)^{\alpha-1}}{\Gamma(\alpha)} f(u, x(u)) d u d s\right]\right\}
\end{aligned}
$$

$\tau_{1}(t)$ and $\tau_{2}(t)$ are defined by (2.15).

Further, we set

$$
\begin{aligned}
\widehat{\tau}_{1}= & \max _{t \in[0,1]}\left|\tau_{1}(t)\right|, \quad \widehat{\tau}_{2}=\max _{t \in[0,1]}\left|\tau_{2}(t)\right| \\
\gamma= & \frac{1}{\left|\delta_{0} b\right| \Gamma(\alpha+1)}\left\{\left(1+\widehat{\tau}_{2}\right)\left|b-b e^{-a} \cos b-a e^{-a} \sin b\right|\right. \\
& +\widehat{\tau}_{1}\left[\xi^{\alpha}\left|b-b e^{-a \xi} \cos b \xi-a e^{-a \xi} \sin b \xi\right|\right. \\
& \left.+\sum_{i=1}^{n}\left|j_{i}\right| \eta_{i}^{\alpha}\left|b-b e^{-a \eta_{i}} \cos b \eta_{i}-a e^{-a \eta_{i}} \sin b \eta_{i}\right|\right] \\
& \left.+\widehat{\tau}_{2} \sum_{i=1}^{k}\left|\lambda_{i}\right| \sigma_{i}^{\alpha}\left|b\left(\sigma_{i}-v_{i}\right)-e^{-a\left(\sigma_{i}-v_{i}\right)} \sin b\left(\sigma_{i}-v_{i}\right)\right|\right\}, \\
\gamma_{1}= & \gamma-\frac{\left|b-b e^{-a} \cos b-a e^{-a} \sin b\right|}{\left|\delta_{0} b\right| \Gamma(\alpha+1)}, \quad a=\frac{\delta_{1}}{2 \delta_{2}}, \quad b=\frac{\sqrt{4 \delta_{0} \delta_{2}-\delta_{1}{ }^{2}}}{2 \delta_{2}} .
\end{aligned}
$$

As before, we can formulate existence results for the problem (1.1)-(1.2) with $\delta_{1}^{2}-4 \delta_{0} \delta_{2}<0$ as follows.

Theorem 5.1 Let $f:[0,1] \times \mathbb{R} \rightarrow \mathbb{R}$ be a continuous function satisfying conditions $\left(\mathrm{A}_{1}\right)$ and $\left(\mathrm{A}_{2}\right)$. Then the problem (1.1)-(1.2), with $\delta_{1}^{2}-4 \delta_{0} \delta_{2}<0$, has at least one solution on $[0,1]$ provided that $\ell \gamma_{1}<1$, where $\gamma$ is given by (5.2).

Theorem 5.2 Assume that $f:[0,1] \times \mathbb{R} \rightarrow \mathbb{R}$ is a continuous function such that $\left(\mathrm{A}_{1}\right)$ is satisfied. Then there exists a unique solution for the problem (1.1)-(1.2), with $\delta_{1}^{2}-4 \delta_{0} \delta_{2}<0$, on $[0,1]$ if $\ell<1 / \gamma$, where $\gamma$ is given by $(5.2)$.

Theorem 5.3 Let $f:[0,1] \times \mathbb{R} \rightarrow \mathbb{R}$ be a continuous function. Further, suppose that $\left(\mathrm{H}_{1}\right)$ and the following condition hold:

$\left(\mathrm{H}_{2}^{\prime \prime}\right)$ There exists a constant $K_{2}>0$ such that $\frac{K_{2}}{\|g\| Q\left(K_{2}\right) \gamma}>1$, where $\gamma$ is defined by (5.2). Then the problem (1.1)-(1.2), with $\delta_{1}^{2}-4 \delta_{0} \delta_{2}<0$, has at least one solution on $[0,1]$.

Example 5.4 Consider the following multi-term fractional differential equation

$$
\left(2^{\mathrm{c}} D^{7 / 3}+2^{\mathrm{c}} D^{4 / 3}+{ }^{\mathrm{c}} D^{1 / 3}\right) x(t)=f(t, x), \quad 0<t<1,
$$

equipped with the boundary conditions (3.8), where

$$
f(t, x)=\frac{L}{3 \sqrt{t^{3}+9}}\left(\cos x+e^{-2 t}\right), \quad L>0 .
$$


Clearly, $\delta_{1}^{2}-4 \delta_{0} \delta_{2}=-4<0$ and $|f(t, x)-f(t, y)| \leq \ell|x-y|$ with $\ell=L / 9$. Using the given values, it is found that $\gamma \approx 0.57912$ and $\gamma_{1} \approx 0.38098$. Further, it is easy to check that $|f(t, x)| \leq \frac{L\left(1+e^{-2 t}\right)}{3 \sqrt{t^{3}+9}}=\vartheta(t)$ and $\ell \gamma_{1}<1$ when $L<23.62329$. As all the conditions of Theorem 5.1 are satisfied, equation (5.3) with the boundary conditions (3.8) has at least one solution on $[0,1]$. On the other hand, since $\ell \gamma<1$ for $L<15.54082$, there exists a unique solution for equation (5.3) with the boundary conditions $(3.8)$ on $[0,1]$ by Theorem 5.2.

\section{Conclusions}

We have derived existence results for a multi-term fractional differential equation associated with different combinations $\left(\delta_{1}^{2}-4 \delta_{0} \delta_{2}>0, \delta_{1}^{2}-4 \delta_{0} \delta_{2}=0\right.$, and $\left.\delta_{1}^{2}-4 \delta_{0} \delta_{2}<0\right)$ of the constants involved in the equation equipped with nonlocal multi-point and multi-strip boundary conditions. Our results are not only new in the given context, but also yield some interesting new results as special cases of the obtained work. For instance, by taking $\lambda_{i}=0, i=i, \ldots, k$ in the results of this paper, we obtain new results for the multi-term fractional differential equation (1.1) associated with the boundary condition of the form: $x(0)=0, x(\xi)=\sum_{i=1}^{n} j_{i} x\left(\eta_{i}\right), x(1)=0$. Our results correspond to those for (1.1) with the nonlocal multi-strip boundary condition: $x(0)=0, x(\xi)=0, x(1)=\sum_{i=1}^{k} \lambda_{i} \int_{v_{i}}^{\sigma_{i}} x(s) d s$ if we fix $j_{i}=0, i=1, \ldots, n$ in the obtained results.

\section{Acknowledgements}

This project was funded by the Deanship of Scientific Research (DSR), King Abdulaziz University, Jeddah, Saudi Arabia under grant no. (KEP-PhD-11-130-39). The authors, therefore, acknowledge with thanks DSR technical and financial support. The authors also thank the reviewers for their constructive remarks on this paper.

\section{Funding}

This project was funded by the Deanship of Scientific Research (DSR), King Abdulaziz University, Jeddah, Saudi Arabia under grant no. (KEP-PhD-11-130-39).

\section{Abbreviations}

Not applicable.

Availability of data and materials

Not applicable

Competing interests

The authors declare that they have no competing interests.

Authors' contributions

Each of the authors, RPA, AA, NA, SKN and BA, contributed equally to each part of this work. All authors read and approved the final manuscript.

\section{Author details}

'Department of Mathematics, Texas A\&M University, Kingsville, USA. ${ }^{2}$ Florida Institute of Technology, Melbourne, USA. ${ }^{3}$ Nonlinear Analysis and Applied Mathematics (NAAM) — Research Group, Department of Mathematics, Faculty of Science, King Abdulaziz University, Jeddah, Saudi Arabia. ${ }^{4}$ Department of Mathematics, Faculty of Science, University of Jeddah, Jeddah, Saudi Arabia. ${ }^{5}$ Department of Mathematics, University of loannina, Ioannina, Greece.

\section{Publisher's Note}

Springer Nature remains neutral with regard to jurisdictional claims in published maps and institutional affiliations.

Received: 11 July 2018 Accepted: 10 September 2018 Published online: 25 September 2018

References

1. Torvik, P.J., Bagley, R.L.: On the appearance of the fractional derivative in the behavior of real materials. J. Appl. Mech. 51, 294-298 (1984)

2. Mainardi, F.: Some basic problems in continuum and statistical mechanics. In: Carpinteri, A., Mainardi, F. (eds.) Fractals and Fractional Calculus in Continuum Mechanics, pp. 291-348. Springer, Berlin (1997)

3. Glöckle, W., Nonnenmacher, T.: A fractional calculus approach to self-similar protein dynamics. Biophys. J. 68, 46-53 (1995)

4. Magin, R.L.: Fractional Calculus in Bioengineering. Begell House Publishers, Danbury (2006) 
5. Sabatier, J., Agrawal, O.P., Machado, J.A.T. (eds.): Advances in Fractional Calculus: Theoretical Developments and Applications in Physics and Engineering. Springer, Dordrecht (2007)

6. Mainardi, F: Fractional Calculus and Waves in Linear Viscoelasticy. World Scientific, Singapore (2010)

7. Herrmann, R.: Fractional Calculus: An Introduction for Physicists. World Scientific, Singapore (2011)

8. Graef, J.R., Kong, L., Kong, Q.: Application of the mixed monotone operator method to fractional boundary value problems. Fract. Differ. Calc. 2, 554-567 (2011)

9. Bai, Z., Sun, W.: Existence and multiplicity of positive solutions for singular fractional boundary value problems. Comput. Math. Appl. 63, 1369-1381 (2012)

10. Ntouyas, S.K., Tariboon, J., Sudsutad, W.: Boundary value problems for Riemann-Liouville fractional differential inclusions with nonlocal Hadamard fractional integral conditions. Mediterr. J. Math. 13, 939-954 (2016)

11. Ahmad, B., Alsaedi, A., Ntouyas, S.K., Tariboon, J.: Hadamard-Type Fractional Differential Equations, Inclusions and Inequalities. Springer, Cham (2017)

12. Agarwal, R.P., Ahmad, B., Alsaedi, A.: Fractional-order differential equations with anti-periodic boundary conditions: a survey. Bound. Value Probl. 2017, 173 (2017)

13. Henderson, J., Luca, R., Tudorache, A.: On a system of fractional differential equations with coupled integral boundary conditions. Fract. Calc. Appl. Anal. 18, 361-386 (2015)

14. Zhang, L., Ahmad, B., Wang, G.: Successive iterations for positive extremal solutions of nonlinear fractional differential equations on a half line. Bull. Aust. Math. Soc. 91, 116-128 (2015)

15. Ahmad, B., Ntouyas, S.K.: Some fractional-order one-dimensional semi-linear problems under nonlocal integral boundary conditions. Rev. R. Acad. Cienc. Exactas Fís. Nat., Ser. A Mat. 110, 159-172 (2016)

16. Ahmad, B., Ntouyas, S.K., Tariboon, J., Alsaedi, A.: A study of nonlinear fractional-order boundary value problem with nonlocal Erdélyi-Kober and generalized Riemann-Liouville type integral boundary conditions. Math. Model. Anal. 22 121-139 (2017)

17. Agarwal, R.P., Ahmad, B., Garout, D., Alsaedi, A.: Existence results for coupled nonlinear fractional differential equations equipped with nonlocal coupled flux and multi-point boundary conditions. Chaos Solitons Fractals 102, 149-161 (2017)

18. Wang, G., Pei, K., Agarwal, R.P., Zhang, L., Ahmad, B.: Nonlocal Hadamard fractional boundary value problem with Hadamard integral and discrete boundary conditions on a half-line. J. Comput. Appl. Math. 343, 230-239 (2018)

19. Ahmad, B., Luca, R.: Existence of solutions for sequential fractional integro-differential equations and inclusions with nonlocal boundary conditions. Appl. Math. Comput. 339, 516-534 (2018)

20. Liu, Y.: Boundary value problems of singular multi-term fractional differential equations with impulse effects. Math. Nachr. 289, 1526-1547 (2016)

21. Ahmad, B., Matar, M.M., El-Salmy, O.M.: Existence of solutions and Ulam stability for Caputo type sequential fractional differential equations of order $\alpha \in(2,3)$. Int. J. Anal. Appl. 15, 86-101 (2017)

22. Stanek, S.: Periodic problem for two-term fractional differential equations. Fract. Calc. Appl. Anal. 20, 662-678 (2017)

23. Alsaedi, A., Alghamdi, N., Agarwal, R.P., Ntouyas, S.K., Ahmad, B.: Multi-term fractional-order boundary-value problems with nonlocal integral boundary conditions. Electron. J. Differ. Equ. 2018, 87 (2018)

24. Kilbas, A.A., Srivastava, H.M., Trujillo, J.J.: Theory and Applications of Fractional Differential Equations. North-Holland Mathematics Studies, vol. 204. Elsevier, Amsterdam (2006)

25. Zhou, Y.: Basic Theory of Fractional Differential Equations. World Scientific, Hackensack (2014)

26. Krasnoselskii, M.A.: Two remarks on the method of successive approximations. Usp. Mat. Nauk 10, 123-127 (1955)

27. Granas, A., Dugundji, J.: Fixed Point Theory. Springer, New York (2003)

\section{Submit your manuscript to a SpringerOpen ${ }^{\circ}$ journal and benefit from:}

- Convenient online submission

- Rigorous peer review

- Open access: articles freely available online

- High visibility within the field

- Retaining the copyright to your article

Submit your next manuscript at $\gg$ springeropen.com 